Check for updates

Cite this: RSC Adv., 2019, 9, 32210

Received 3rd September 2019 Accepted 2nd October 2019

DOI: 10.1039/c9ra07050h

rsc.li/rsc-advances

\section{Suzuki coupling-based synthesis of VATPase inhibitor archazolid natural product derived fragments $\dagger$}

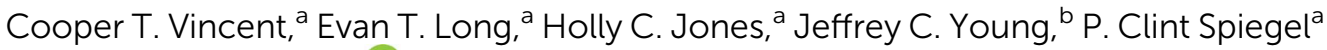 \\ and Gregory W. O'Neil (DD *a
}

An archazolid natural product fragment that displays dose-dependent inhibition of the vacuolar-type ATPase (VATPase) has been synthesized by a high-yielding Suzuki coupling of two complex subunits. Similarly, a further simplified fragment was prepared and evaluated for VATPase inhibitory activity. This compound did inhibit the VATPase, as evidenced by growth inhibition of etiolated Arabidopsis seedlings, however at approximately $10 \times$ lower potency than the more complex fragment. Cyclooxygenase (COX) enzyme inhibition was not observed for either fragment.

\section{Introduction}

Recently our group reported the synthesis of an archazolid natural product fragment (1) that, after removal of the TBS protecting groups, displayed dose-dependent inhibition of the vacuolar-type ATPase (VATPase). ${ }^{1}$ The VATPase has continued to emerge as a promising therapeutic target associated with several severe forms of cancer, as evidenced by a number of recent studies involving the archazolids. ${ }^{2-10}$ Key to our synthesis of $\mathbf{1}$ was a high-yielding Stille coupling between iodide 2 and stannane 3 to complete the conjugated $Z, Z, E$-triene (Scheme 1). ${ }^{11}$ This particular triene is unique to the archazolids and happens to link two important pharmacophoric elements, the C7- and C15 hydroxyl groups. ${ }^{12}$

While promising, others have commented on the drawbacks of Stille couplings due to the toxicity associated with organotin compounds..$^{13,14}$ In line with these recommendations, we set out to investigate a tin-free synthesis of our VATPase inhibitor lead compound. Herein we report a similarly effective Suzuki coupling strategy to synthesize compound $\mathbf{1}$, in which tin has been replaced by a boronic acid derivative. We argue that this new approach represents to date the most direct, convergent, and a greener method for synthesizing the important triene region of this exciting class of compounds.

${ }^{a}$ Department of Chemistry, Western Washington University, Bellingham, WA, USA 98229. E-mail: oneilg@wwu.edu

${ }^{b}$ Department of Biology, Western Washington University, Bellingham, WA, USA 98229 $\dagger$ Electronic supplementary information (ESI) available: Copies of NMR spectra for compounds $1,2,4,7,8,10,13-15$, and 17-20. VATPase and COX assay procedures and results. See DOI: $10.1039 / \mathrm{c} 9 \mathrm{ra} 07050 \mathrm{~h}$

\section{Results and discussion}

Tin featured not only in our Stille coupling, but also in our synthesis of vinyl iodide $2,{ }^{1}$ which was prepared by palladium catalyzed hydrostannylation followed by iododestannylation (Scheme 2). To circumvent the use of tin in this instance, we opted instead to carry the TMS-protected alkynyl phosphonate 4, prepared in a single step from known Weinreb amide $5,{ }^{15}$ into a Horner-Wadsworth-Emmons (HWE) olefination ${ }^{16}$ with aldehyde $6 .{ }^{17}$ This reaction delivered ketone 7 in $72 \%$ yield and $>10: 1 E: Z$ selectivity by NMR analysis. The ketone in 7 was diastereoselectively reduced with $\mathrm{NaBH}_{4}$ and the resulting hydroxyl converted to the corresponding methyl ether by deprotonation with LiHMDS and alkylation with methyl iodide. Removal of the TMS group $\left(\mathrm{K}_{2} \mathrm{CO}_{3} / \mathrm{MeOH}\right)$ revealed the terminal<smiles>CCCOCC(C)/C=C\C=C(/C)C(C)C(C)OCC</smiles>
$\mathrm{Pd}\left(\mathrm{PPh}_{3}\right)_{4}, \mathrm{CuTC}$,
$\left[\mathrm{Ph}_{2} \mathrm{PO}_{2}\right]\left[\mathrm{NBu}_{4}\right]$

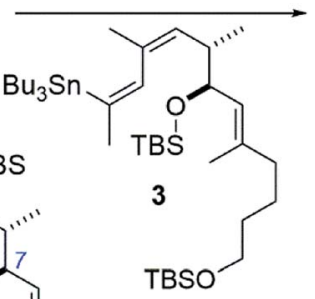<smiles>COc1ccccc1-c1ccccc1</smiles>
TBSO

O'Neil et al. Synlett 2017, 28, 1101.

Scheme 1 Previous synthesis of archazolid-based VATPase inhibitor compound 1 by Stille coupling 
Previous synthesis (O'Neil et al. Synlett 2017, 28, 1101):

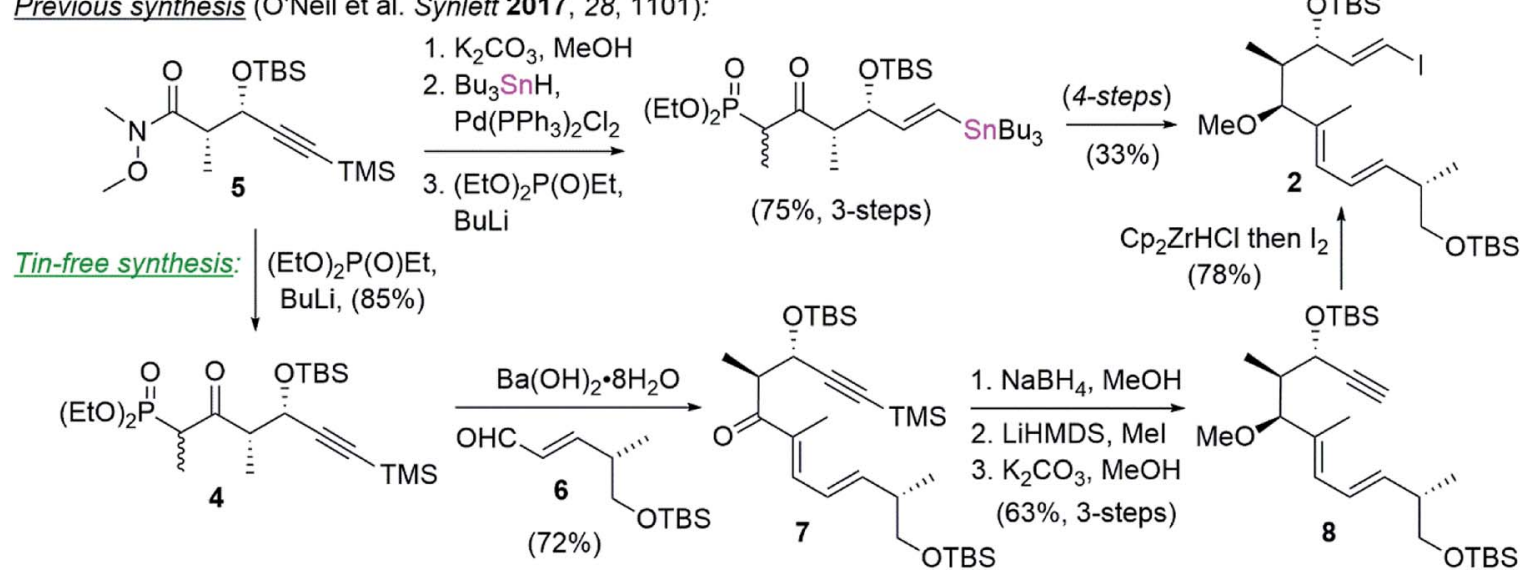

Scheme 2 An alternative tin-free synthesis of vinyl iodide 2.

alkyne in $\mathbf{8}$ and set the stage for vinyl iodide installation by treatment with Schwartz's reagent and trapping with iodine. ${ }^{18}$ In this way, vinyl iodide coupling partner 2 was completed in comparable yields and one fewer step from compound 4 than had previously been described (i.e. 6-steps and 36\% overall yield vs. 7 -steps and $25 \%$ yield) while also avoiding the use of tin.

Adapting our vinyl stannane synthesis to incorporate a vinyl boronic ester was possible from advanced vinyl iodide 9 by trapping with $\mathrm{B}\left({ }^{\mathrm{i}} \mathrm{PrO}\right)_{3}$ rather than $\mathrm{Bu}_{3} \mathrm{SnCl}$ after lithiumhalogen exchange (Scheme 3). ${ }^{19}$ Treatment with pinacol then allowed for isolation of vinyl pinacol boronate 10 in $93 \%$ yield. In an attempt to reduce the step count of our vinyl boronic ester synthesis, we became interested in evaluating a potential boronWittig reaction. This chemistry was pioneered by Endo and coworkers $^{\mathbf{2 0}}$ and more recently has been expanded upon by the Morken group. ${ }^{21,22}$ To that end, intermediate 11 was acylated with phosphonate acid $12{ }^{23}$ using DCC, giving ester 13 in $72 \%$ yield. Removal of the PMB protecting group and oxidation of the resulting primary hydroxyl then allowed for an intramolecular HWE reaction. It was found that $\mathrm{Ba}(\mathrm{OH})_{2}$ gave the best overall yields of lactone 14, with some other reagents (e.g. KHMDS, $\mathrm{DBU} / \mathrm{LiCl}$ ) leading primarily to elimination. Reduction of $\mathbf{1 4}$ with DIBAL-H produced the corresponding lactol, that we thought could be converted directly to the desired $E$-vinyl boronic ester 15 by treatment with the lithium anion of a geminal bis(boronate). However, using Morken's procedure ${ }^{21}$ for the conversion of aldehydes to tri-substituted vinyl pinacol boronates (LiTMP, $-78{ }^{\circ} \mathrm{C}$ ) using 1,1-bis(pinacolboronato) ethane, no reaction was observed.

We reasoned that the lactol was less reactive than an aldehyde in these reactions, and therefore repeated the experiment allowing the reaction to slowly warm to room temperature. Under these conditions, the vinyl boronate ester product 15 was indeed obtained, albeit as a $3: 1$ mixture of geometrical isomers

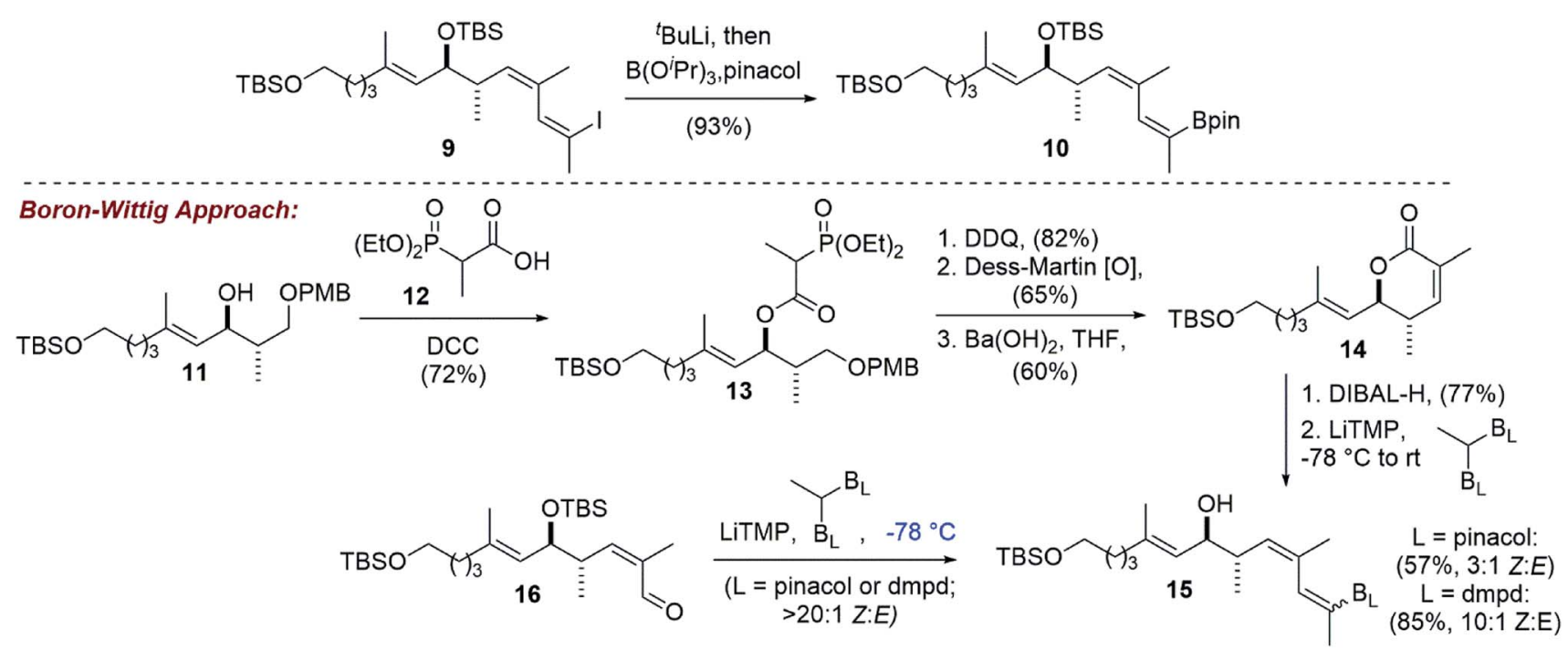

Scheme 3 Vinyl boronic ester synthesis by lithium-halogen exchange and an alternative boron-Wittig approach that gave primarily the undesired Z-vinyl boronate. 


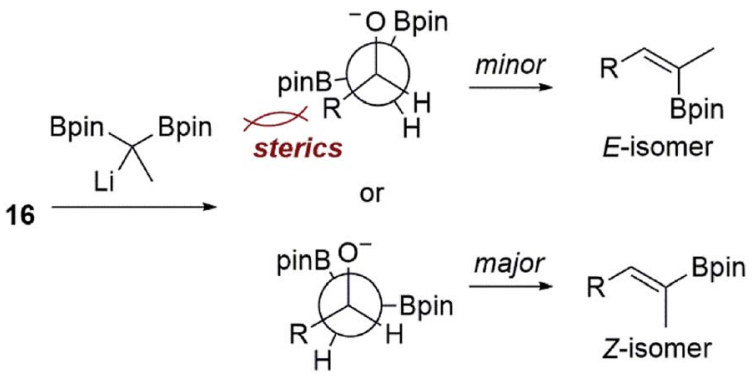

Fig. 1 Possible stereochemical rationale for the $E: Z$ selectivity observed in our boron-Wittig reactions with the lactol derived from 13 or alkdehyde 15 shown in Scheme 3.

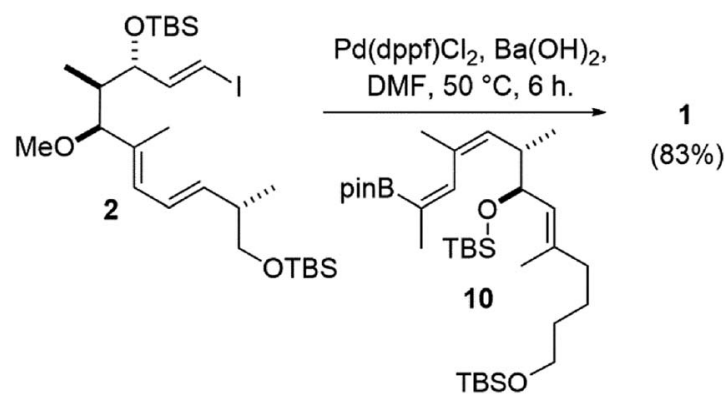

Scheme 4 Synthesis of the archazolid conjugated triene by Suzuki coupling.

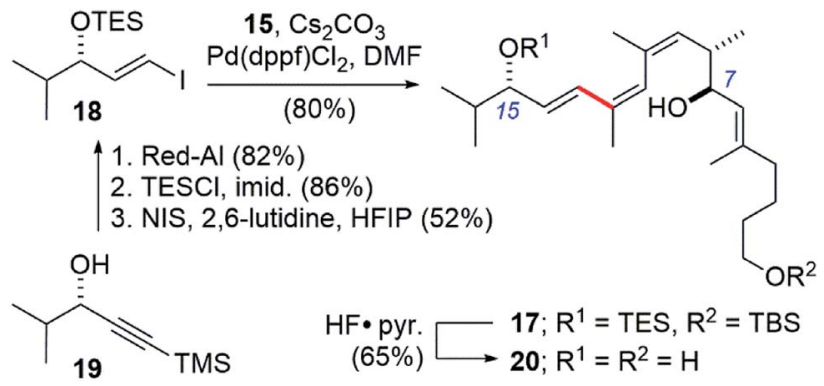

Scheme 5 Synthesis of a simplified archazolid fragment 20 containing the pharmacophoric C7- and C15-hydroxyls linked by the Z,Z,E-triene.

by NMR analysis. Using 2D-NMR techniques (e.g. COSY and NOESY), it was determined that the major product from this reaction was the undesired $Z$-vinyl boronate. Morken's group had reported that in some cases switching to the dimethylpentanediolato (dpmd) diboronate led to a reversal of stereoselectivity when compared to the pinacol diboronate. ${ }^{21}$ Unfortunately, the reaction with 1,1-bis(dimethylpentanediolato)ethane was even more selective for the undesired $Z$-vinyl boronic ester (10:1 $Z: E$ ). When using aldehyde 16, reactions that proceeded at the colder temperatures $\left(-78{ }^{\circ} \mathrm{C}\right)$ prescribed by Endo and Morken, ${ }^{20,21}$ both diboronates gave exclusively the undesired $Z$-vinyl boronic ester products. We can perhaps rationalize the stereochemical outcome of these reactions by considering a steric clash between the $\mathrm{R}$ group from the substrate and one of the boronic esters after addition of the lithiated diboronate and upon rotation into a conformation with the O- and B-groups syn-coplanar that is required for elimination (Fig. 1). ${ }^{\mathbf{2 0 , 2 2}}$

Nonetheless, the $E / Z$ isomers from the boron-Wittig reactions were separable by chromatography on silica. Combined with our lithium-halogen exchange approach, sufficient quantities of the desired $Z, E$-boronic esters $\mathbf{1 0}$ and $\mathbf{1 5}$ were obtained to then investigate a Suzuki coupling with vinyl iodide $\mathbf{2 .}^{\mathbf{1}}$ Gratifyingly, it was found that the use of $10 \mathrm{~mol} \% \operatorname{Pd}(\mathrm{dppf}) \mathrm{Cl}_{2}$ and $\mathrm{Ba}(\mathrm{OH})_{2}$ as base $\mathrm{b}^{24}$ in $\mathrm{DMF}$ at $50{ }^{\circ} \mathrm{C}$ for $6 \mathrm{~h}$ gave the coupled product 1 in comparable yield to what was previously obtained from a Stille coupling ${ }^{\mathbf{1}}$ ( $83 \%$ for the Suzuki vs. $82 \%$ from Stille coupling, Scheme 4).

We also took this opportunity to prepare a further simplified archazolid fragment 17 (Scheme 5). The requisite vinyl iodide 18 was prepared from alkynol 19, available in by enantioselective Noyori reduction $^{25}$ of the corresponding alkynone. ${ }^{26}$ Red-Al reduction of the alkyne in 19 to the trans-alkene, TES protection of the alcohol, and iododesilylation of the TMS group gave 18 in $37 \%$ yield for the three steps. The Suzuki coupling with boronic ester $\mathbf{1 0}$ again proceeded efficiently, in this case using $\mathrm{Pd}(\mathrm{dppf}) \mathrm{Cl}_{2}$ and $\mathrm{Cs}_{2} \mathrm{CO}_{3}$ as base in DMF at room temperature, ${ }^{27}$ giving the coupled product $\mathbf{1 7}$ in $80 \%$ yield.

Given that 17 contained an intact $\mathrm{C} 7-\mathrm{C} 15$ region of the archazolids, we suspected this compound might exhibit VATPase inhibitory activity and opted to assay the desilylated compound 20 using our Arabidopsis-based method. ${ }^{28,29}$ As shown in Fig. 2, while 20 did exhibit dose dependent growth inhibition of etiolated Arabidopsis as an indicator for impaired VATPase function, its activity was significantly lower $(\sim 10 \times$ less potent) than compound 1 . This data suggests that while the C7C15 segment of the archazolids is important for VATPase binding, additional functionality present in compound $\mathbf{1}$ also contributes to its effectiveness as a VATPase inhibitor.

Compound 20 is similar the predicted cyclooxygenase (COX) enzyme inhibitor ArcA-1 ${ }^{30}$ (Fig. 3). When tested using a commercial COX screening assay for inhibition of COX-1 and COX- $2,{ }^{31}$ no measurable inhibition was observed at concentrations up to $200 \mu \mathrm{M}$. This was also true for compound 1, which we previously postulated could be due to its large size and/or lack of a carboxylic acid terminus. ${ }^{1}$ The latter might also apply to compound 20, however neither rationale would explain the modest reported COX inhibitory activity of archazolid $\mathrm{A}^{30}$ which is both sterically large and lacks a carboxylic acid.

\section{Conclusions}

We have devised and executed a high-yielding Suzuki couplingbased synthesis of the challenging yet biologically important conjugated triene region of the archazolid natural products. The vinyl iodide synthesis reported here represents an improvement over our previous approach in terms of step count, yield, and avoidance of hazardous tin compounds. Two different vinyl boronic ester syntheses were investigated. Ultimately it was found that while a boron-Wittig reaction did give some of the desired compound, the majority of product obtained was the undesired geometrical isomer. The isomers 


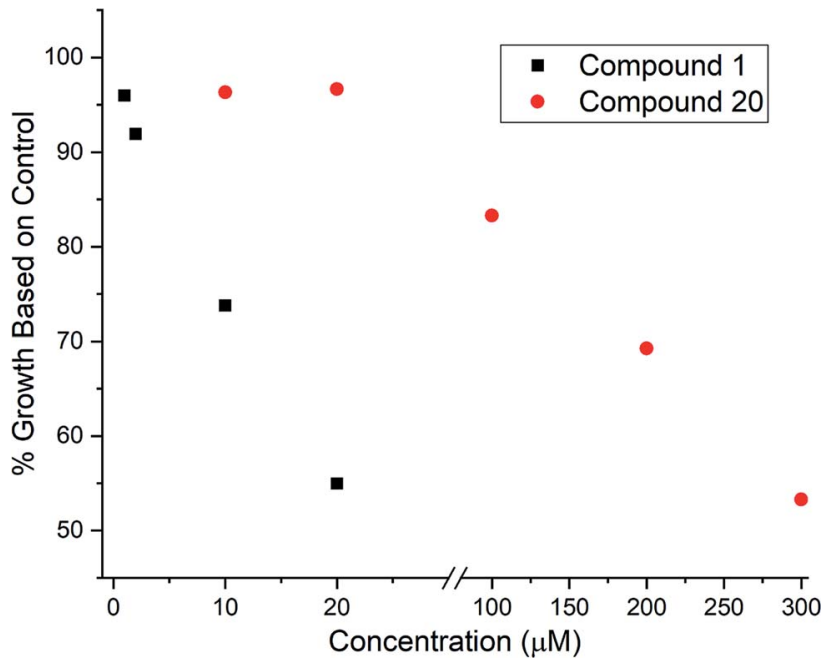

Fig. 2 VATPase assay results for compound 1 (after TBS removal) and compound 20 where \% growth relative to control represents the average hypocotyl length for 40-60 Arabidopsis seedlings at the concentrations indicated relative to the average hypocotyl length of Arabidopsis seedlings grown in the absence of any inhibitors.

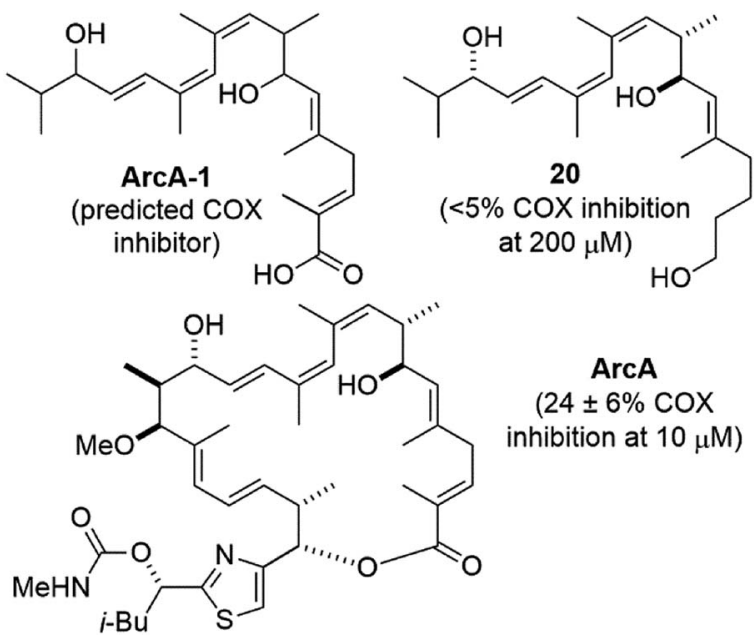

Fig. 3 Structures and cyclooxygenase (COX) inhibitory activity of proposed COX inhibitor ArcA-1, ${ }^{30}$ archazolid A (ArcA), and compound 20.

were, however, separable and combined with an alternative iodo-Wittig followed by lithium-halogen exchange-based synthesis, sufficient amounts of the correct boronic ester isomer were obtained to then conduct Suzuki couplings. In this way, an archazolid natural product fragment 1 that had previously exhibited dose dependent VATPase inhibition along with a further simplified fragment $\mathbf{2 0}$ were synthesized. Compound 20 also showed dose dependent VATPase inhibition in our Arabidopsis VATPase assay, however with approximately $10 \times$ less potency than compound 1 . The assay results support the importance of the C7-C15 region of the archazolids for VATPase inhibitory activity, but suggest additional functionality is needed for efficient VATPase inhibition. Compound 20 was also assayed for COX inhibitory activity based on its similarity to a predicted COX inhibitor. However, like compound 1, in our assay no measurable COX inhibition was observed for compound 20. Further studies are underway to better understand the interactions of compounds $\mathbf{1}$ and 20 with VATPase and COX enzymes toward the design of more efficient inhibitors with therapeutic potential.

\section{Experimental section}

\section{General information}

All reactions were carried out under $\mathrm{N}_{2}$ in flame-dried glassware. IR: Nicolet iS10 spectrometer, wavenumbers $(\tilde{v})$ in $\mathrm{cm}^{-1}$. The solvents used were dried by passing the solvent through a column of activated alumina under nitrogen immediately prior to use. All reagents were purchased and used as received unless otherwise mentioned. All TLC analysis used $0.25 \mathrm{~mm}$ silica layer fluorescence $\mathrm{UV}_{254}$ plates. Flash chromatography: SilaCycle silica gel P60 (230-400 mesh). NMR: spectra were recorded on a Varian Mercury 300, or Inova 500 spectrometer in the solvents indicated; chemical shifts $(\delta)$ are given in ppm, coupling constants $(J)$ in $\mathrm{Hz}$. The solvent signals were used as references $\left(\mathrm{C}_{6} \mathrm{D}_{6}: \delta_{\mathrm{C}} \equiv 128.0 \mathrm{ppm}\right.$; residual $\mathrm{C}_{6} \mathrm{H}_{6}$ in $\mathrm{C}_{6} \mathrm{D}_{6}: \delta_{\mathrm{H}} \equiv$ $7.16 \mathrm{ppm} ; \mathrm{CDCl}_{3}: \delta_{\mathrm{C}} \equiv 77.0 \mathrm{ppm}$; residual $\mathrm{CHCl}_{3}$ in $\mathrm{CDCl}_{3}: \delta_{\mathrm{H}}$ $\equiv 7.26 \mathrm{ppm})$.

Diethyl ((4S,5S)-5-((tert-butyldimethylsilyl)oxy)-4-methyl-3oxo-7-(trimethylsilyl)hept-6-yn-2-yl)phosphonate (4). To a solution of diethyl ethylphosphonate $(1.02 \mathrm{~g}, 6.15 \mathrm{mmol})$ in THF (14 $\mathrm{mL})$ at $-78^{\circ} \mathrm{C}$ was added a solution of $n$-butyllithium $(2.46 \mathrm{~mL}$, $2.5 \mathrm{M}$ in hexanes) and the mixture was stirred for $20 \mathrm{~min}$. Weinreb amide $5{ }^{15}$ (1.0 g, $2.8 \mathrm{mmol}$ ) was then added and the reaction was stirred for 30 min before quenching with aq. $\mathrm{NH}_{4} \mathrm{Cl}(50 \mathrm{~mL})$ and extracting with EtOAc $(2 \times 50 \mathrm{~mL})$. The combined organic extracts were dried over $\mathrm{MgSO}_{4}$, filtered, and concentrated in vacuo. Purification by flash column chromatography on silica $(1: 1$ hexanes : ethyl acetate) afforded phosponate $4(1.1 \mathrm{~g}, 85 \%)$ as an oil.

Spectral data for the major diastereomer. IR (ATR): 3036, 29 078, 1710, 1635, 1460, 1364, 1191, 1077, 910, $778 \mathrm{~cm}^{-1} .{ }^{1} \mathrm{H}$ NMR $\left(500 \mathrm{MHz}, \mathrm{CDCl}_{3}\right): \delta 4.24(\mathrm{~d}, J=8.1 \mathrm{~Hz}, 1 \mathrm{H}), 4.13(\mathrm{q}, J=$ $6.4 \mathrm{~Hz}, 2 \mathrm{H}), 4.11(\mathrm{q}, J=6.4 \mathrm{~Hz}, 2 \mathrm{H}), 3.70\left(\mathrm{dq}, J_{\mathrm{HP}}=26.0 \mathrm{~Hz}, J_{\mathrm{HH}}\right.$ $=6.7 \mathrm{~Hz}, 1 \mathrm{H}), 3.30(\mathrm{~m}, 1 \mathrm{H}), 1.33(\mathrm{~d}, J=7.0 \mathrm{~Hz}, 3 \mathrm{H}), 1.32(\mathrm{t}, J=$ $7.0 \mathrm{~Hz}, 3 \mathrm{H}), 1.31(\mathrm{t}, J=7.0 \mathrm{~Hz}, 3 \mathrm{H}), 1.14(\mathrm{~d}, J=6.6 \mathrm{~Hz}, 3 \mathrm{H}), 0.89$ (s, 9H), $0.14(\mathrm{~s}, 3 \mathrm{H}), 0.13(\mathrm{~s}, 9 \mathrm{H}), 1.11(\mathrm{~s}, 3 \mathrm{H}) .{ }^{13} \mathrm{C}$ NMR $(125$ $\left.\mathrm{MHz}, \mathrm{CDCl}_{3}\right): \delta 207.1,105.0,91.2,65.5,62.5\left(\mathrm{t}, J_{\mathrm{CP}}=6.4 \mathrm{~Hz}, 1 \mathrm{C}\right)$, $54.2,48.4,47.5,25.70,25.67,18.2,16.4\left(\mathrm{t}, J_{\mathrm{CP}}=5.4 \mathrm{~Hz}, 1 \mathrm{C}\right), 13.2$, $10.6\left(J_{\mathrm{CP}}=6.2 \mathrm{~Hz}, 1 \mathrm{C}\right),-0.3,-0.4,-4.5,-5.1$. HRMS-TOF (ESI+) calcd for $\mathrm{C}_{21} \mathrm{H}_{43} \mathrm{O}_{5} \mathrm{PSi}_{2} \mathrm{Na}^{+}(\mathrm{M}+\mathrm{Na})$ : 485.2284. Found 485.2279 .

$(5 S, 6 S, 8 E, 10 E, 12 S)-2,2,3,3,6,8,12,15,15,16,16-$ Undecamethyl5-((trimethylsilyl)ethynyl)-4,14-dioxa-3,15-disilaheptadeca-8,10dien-7-one (7). $\mathrm{Ba}(\mathrm{OH})_{2} \cdot 8 \mathrm{H}_{2} \mathrm{O}(1.8 \mathrm{~g}, 5.73 \mathrm{mmol})$ was first activated by heating under vacuum at $130{ }^{\circ} \mathrm{C}$ for $1.5 \mathrm{~h}$ and then cooled to room temperature before adding THF $(10 \mathrm{~mL})$ and phosphonate $4(1.1 \mathrm{~g}, 2.4 \mathrm{mmol})$ and the mixture was stirred for $30 \mathrm{~min}$. Aldehyde $\mathbf{6}^{17}(0.66 \mathrm{~g}, 2.9 \mathrm{mmol})$ was then added and the reaction was stirred for $8 \mathrm{~h}$ before quenching with aq. $\mathrm{NaHCO}_{3}$ 
$(30 \mathrm{~mL})$ and extracting with MTBE $(2 \times 30 \mathrm{~mL})$. The combined organic extracts were dried over $\mathrm{MgSO}_{4}$, filtered, and concentrated in vacuo. Purification by flash column chromatography on silica (20:1 to $10: 1$ hexanes : ethyl acetate) afforded 7 $(0.93 \mathrm{~g}, 72 \%)$ as an oil.

$[\alpha]_{\mathrm{D}}^{20}=-2.8\left(c 1.0, \mathrm{CH}_{2} \mathrm{Cl}_{2}\right)$. IR (ATR) 3064, 2903, 1735, 1640, 1350, 1194, 1067, 910, $730 \mathrm{~cm}^{-1} \cdot{ }^{1} \mathrm{H}$ NMR (500 MHz, $\mathrm{CDCl}_{3}$ ): $\delta 7.07(\mathrm{~d}, J=10.9 \mathrm{~Hz}, 1 \mathrm{H}), 6.48(\mathrm{ddd}, J=15.0,10.9,1.1 \mathrm{~Hz}, 1 \mathrm{H})$, $6.08(\mathrm{dd}, J=15.2,7.5 \mathrm{~Hz}, 1 \mathrm{H}), 4.38$ (d, $J=10.5 \mathrm{~Hz}, 1 \mathrm{H}), 3.60-$ $3.47(\mathrm{~m}, 3 \mathrm{H}), 2.50(\mathrm{p}, J=6.6 \mathrm{~Hz}, 1 \mathrm{H}), 1.88(\mathrm{~s}, 3 \mathrm{H}), 1.15(\mathrm{~d}, J=$ $7.0 \mathrm{~Hz}, 3 \mathrm{H}), 1.06$ (d, $J=7.0 \mathrm{~Hz}, 3 \mathrm{H}), 0.91$ (s, 9H), 0.89 (s, 9H), 0.14 (s, 3H), 0.11 (s, 3H), 0.06 (s, 9H), 0.05 (s, 3H), 0.04 (s, 3H). ${ }^{13} \mathrm{C}$ NMR (125 MHz, $\mathrm{CDCl}_{3}$ ): $\delta{ }^{13} \mathrm{C} \mathrm{NMR}\left(126 \mathrm{MHz}, \mathrm{CDCl}_{3}\right.$ ) $\delta$ 203.5, 146.2, 139.3, 134.7, 126.3, 106.1, 90.1, 67.4, 65.7, 46.8, $40.3,25.9,25.8,18.3,16.3,14.6,11.8,-0.4,-4.5,-5.1,-5.3$. HRMS-TOF (ESI+) calcd for $\mathrm{C}_{29} \mathrm{H}_{56} \mathrm{O}_{3} \mathrm{Si}_{3} \mathrm{Na}^{+}(\mathrm{M}+\mathrm{Na}): 559.3435$. Found 559.3428.

$(5 S, 6 R, 7 S, 8 E, 10 E, 12 S)-2,2,3,3,6,8,12,15,15,16,16-$ Undecamethyl-5-((trimethylsilyl)ethynyl)-4,14-dioxa-3,15-dis-

ilaheptadeca-8,10-dien-7-ol. To a solution of ketone $7(0.2 \mathrm{~g}$, $0.37 \mathrm{mmol})$ in $\mathrm{MeOH}(1.0 \mathrm{~mL})$ and THF $(1.0 \mathrm{~mL})$ at $0{ }^{\circ} \mathrm{C}$ was added $\mathrm{NaBH}_{4}(54 \mathrm{mg}, 1.44 \mathrm{mmol})$ and the mixture was stirred and allowed to slowly warm to room temperature for $2 \mathrm{~h}$. The reaction was quenched with brine $(15 \mathrm{~mL})$ and extracted with MTBE $(2 \times 15 \mathrm{~mL})$. The combined organic extracts were dried over $\mathrm{MgSO}_{4}$, filtered, and concentrated in vacuo. Purification by flash column chromatography on silica (20:1 to $10: 1$ hexanes : ethyl acetate) afforded the alcohol $(0.93 \mathrm{~g}, 72 \%)$ as an oil.

$[\alpha]_{\mathrm{D}}^{20}=-4.6\left(c\right.$ 1.0, $\mathrm{CH}_{2} \mathrm{Cl}_{2}$ ). IR (ATR) 3201, 2958, 2930, 2859, 1447, 1371, 1308, 1221, 1143, 1085, 1024, 755, $699 \mathrm{~cm}^{-1} .{ }^{1} \mathrm{H}$ NMR (500 MHz, $\left.\mathrm{C}_{6} \mathrm{D}_{6}\right): \delta 6.37$ (ddd, $\left.J=15.2,10.8,1.2 \mathrm{~Hz}, 1 \mathrm{H}\right)$, $6.13(\mathrm{dd}, J=11.0,1.5 \mathrm{~Hz}, 1 \mathrm{H}), 5.58(\mathrm{dd}, J=15.2,7.5 \mathrm{~Hz}, 1 \mathrm{H})$, $5.03(\mathrm{~d}, J=2.5 \mathrm{~Hz}, 1 \mathrm{H}), 4.14(\mathrm{dd}, J=9.0,2.8 \mathrm{~Hz}, 1 \mathrm{H}), 3.49(\mathrm{dd}, J$ $=9.6,5.9 \mathrm{~Hz}, 1 \mathrm{H}), 3.38(\mathrm{dd}, J=9.6,6.8 \mathrm{~Hz}, 1 \mathrm{H}), 2.41$ (hept, $J=$ $6.7 \mathrm{~Hz}, 1 \mathrm{H}), 2.12$ (d, $J=2.9 \mathrm{~Hz}, \mathrm{O}-\mathrm{H}), 2.00(\mathrm{dqd}, J=9.3,6.9$, $2.5 \mathrm{~Hz}, 1 \mathrm{H}), 1.64(\mathrm{~s}, 3 \mathrm{H}), 1.08$ (d, $J=7.0 \mathrm{~Hz}, 3 \mathrm{H}), 1.04$ (d, $J=$ $6.8 \mathrm{~Hz}, 3 \mathrm{H}), 1.02(\mathrm{~s}, 9 \mathrm{H}), 0.97(\mathrm{~s}, 9 \mathrm{H}) 0.29(\mathrm{~s}, 3 \mathrm{H}), 0.19(\mathrm{~s}, 3 \mathrm{H})$, 0.17 (s, 9H), 0.05 (s, 6H). ${ }^{13} \mathrm{C}$ NMR (125 MHz, $\left.\mathrm{C}_{6} \mathrm{D}_{6}\right): \delta$ 137.3, 137.0, 126.1, 107.6, 90.0, 79.6, 68.2, 65.0, 43.0, 40.2, 31.9, 26.1, 26.0, 23.0, 18.52, 18.45, 16.9, 14.3, 11.6, 11.5, -0.1, -4.2, -5.0, -5.20, -5.24. HRMS-TOF (ESI+) calcd for $\mathrm{C}_{29} \mathrm{H}_{58} \mathrm{O}_{3} \mathrm{Si}_{3} \mathrm{Na}^{+}(\mathrm{M}+$ $\mathrm{Na})$ : 561.3591. Found 561.3591.

$(6 S, 7 E, 9 E, 11 S, 12 R, 13 S)$-13-Ethynyl-11-methoxy2,2,3,3,6,10,12,15,15,16,16-undecamethyl-4,14-dioxa-3,15-

disilaheptadeca-7,9-diene (8). To a solution of the intermediate alcohol in THF $(3.7 \mathrm{~mL})$ at $-78{ }^{\circ} \mathrm{C}$ was added a solution of LiHMDS (0.44 $\mathrm{mL}, 1.0 \mathrm{M}$ in toluene) at $-78{ }^{\circ} \mathrm{C}$ and the mixture was stirred for $20 \mathrm{~min}$. Iodomethane $(50 \mu \mathrm{L}, 0.74 \mathrm{mmol})$ was then added and the reaction was allowed to slowly warm to room temperature over $15 \mathrm{~h}$ before quenching with $\mathrm{NaHCO}_{3}(30$ $\mathrm{mL})$ and extracting with $\operatorname{MTBE}(2 \times 30 \mathrm{~mL})$. The combined organic extracts were dried over $\mathrm{MgSO}_{4}$, filtered, and concentrated in vacuo. The crude methyl ether product was redissolved in $\mathrm{MeOH}(3.0 \mathrm{~mL})$ and $\mathrm{THF}(1.0 \mathrm{~mL})$ and the solution was cooled to $0{ }^{\circ} \mathrm{C}$ before adding $\mathrm{K}_{2} \mathrm{CO}_{3}(0.1 \mathrm{~g}, 0.72 \mathrm{mmol})$ and the mixture was slowly warmed to room temperature over $6 \mathrm{~h}$. The reaction was quenched with aq. $\mathrm{NH}_{4} \mathrm{Cl}(50 \mathrm{~mL})$ and extracted with MTBE $(2 \times 30 \mathrm{~mL})$. The combined organic extracts were dried over $\mathrm{MgSO}_{4}$, filtered, and concentrated in vacuo. Purification by flash column chromatography on silica (20:1 to $10: 1$ hexanes : ethyl acetate) afforded 8 (0.11 g, 63\% 3-steps) as an oil.

$[\alpha]_{\mathrm{D}}^{20}=-3.4\left(c 1.0, \mathrm{CH}_{2} \mathrm{Cl}_{2}\right)$. IR (ATR) 2958, 2930, 2859, 1447, $1371,1308,1221,1143,1085,1024,755,699 \mathrm{~cm}^{-1} \cdot{ }^{1} \mathrm{H}$ NMR $(500$ $\left.\mathrm{MHz}, \mathrm{C}_{6} \mathrm{D}_{6}\right): \delta 6.39(\mathrm{dd}, J=15.2,10.8 \mathrm{~Hz}, 1 \mathrm{H}), 6.09(\mathrm{~d}, J=$ $10.0 \mathrm{~Hz}, 1 \mathrm{H}), 5.55$ (dd, $J=15.2,7.6 \mathrm{~Hz}, 1 \mathrm{H}), 5.22(\mathrm{t}, J=2.0 \mathrm{~Hz}$, $1 \mathrm{H}), 3.53(\mathrm{~d}, J=10.0 \mathrm{~Hz}, 1 \mathrm{H}), 3.46(\mathrm{dd}, J=9.7,6.1 \mathrm{~Hz}, 1 \mathrm{H}), 3.37$ (dd, $J=9.7,6.8 \mathrm{~Hz}, 1 \mathrm{H}), 3.09(\mathrm{~s}, 3 \mathrm{H}), 2.40(\mathrm{p}, J=6.9 \mathrm{~Hz}, 1 \mathrm{H})$, $2.03(\mathrm{~d}, J=2.1 \mathrm{~Hz}, 1 \mathrm{H}), 2.02(\mathrm{~m}, 1 \mathrm{H}), 1.59(\mathrm{~d}, J=1.3 \mathrm{~Hz}, 3 \mathrm{H})$, $1.12(\mathrm{~d}, J=6.9 \mathrm{~Hz}, 3 \mathrm{H}), 1.04(\mathrm{~s}, 9 \mathrm{H}), 1.02(\mathrm{~d}, J=6.7 \mathrm{~Hz}, 3 \mathrm{H}), 0.97$ (s, 9H), $0.29(\mathrm{~s}, 3 \mathrm{H}), 0.18(\mathrm{~s}, 3 \mathrm{H}), 0.04(\mathrm{~s}, 6 \mathrm{H}) .{ }^{13} \mathrm{C}$ NMR (126 $\left.\mathrm{MHz}, \mathrm{C}_{6} \mathrm{D}_{6}\right) \delta 137.3,133.6,130.3,125.5,87.6,85.7,72.3,67.8$, 61.8, 55.2, 42.7, 39.9, 25.7, 18.2, 18.1, 16.4, 10.3, 10.0, -4.6, $-5.5,-5.57,-5.59$. HRMS-TOF (ESI+) calcd for $\mathrm{C}_{27} \mathrm{H}_{52} \mathrm{O}_{3} \mathrm{Si}_{2} \mathrm{Na}^{+}$ $(\mathrm{M}+\mathrm{Na}):$ 503.3353. Found 503.3354.

$(6 S, 7 E, 9 E, 11 S, 12 R, 13 S)-13-((E)-2-I o d o v i n y l)-11-m e t h o x y-$ 2,2,3,3,6,10,12,15,15,16,16-undecamethyl-4,14-dioxa-3,15-

disilaheptadeca-7,9-diene (2). To a solution of alkyne 8 (0.1 g, $0.21 \mathrm{mmol})$ in THF $(2.0 \mathrm{~mL})$ was added zirconocene hydrochloride (30 mg, $0.1 \mathrm{mmol}$ ) and the mixture was stirred at room temperature for $20 \mathrm{~min}$ before adding additional zirconocene hydrochloride (30 mg, $0.1 \mathrm{mmol})$. After another $20 \mathrm{~min}$ at room temperature, iodine ( $66 \mathrm{mg}, 0.26 \mathrm{mmol}$ ) was added and the solution was stirred for $30 \mathrm{~min}$. The reaction was then diluted with hexanes $(10 \mathrm{~mL})$ and filtered through a pad of celite. The resulting solution was washed with sat. aq. $\mathrm{Na}_{2} \mathrm{~S}_{2} \mathrm{O}_{3}(2 \times 15 \mathrm{~mL})$ and brine $(15 \mathrm{~mL})$. The organic phase was dried over $\mathrm{MgSO}_{4}$, filtered, and concentrated in vacuo. Purification by flash column chromatography on silica (10: 1 hexanes : ethyl acetate) afforded $2(0.1 \mathrm{~g}, 78 \%)$ as an oil.

$[\alpha]_{\mathrm{D}}^{20}=-7.8\left(c 1.0, \mathrm{CH}_{2} \mathrm{Cl}_{2}\right)$. IR (ATR) 2955, 2928, 2865, 1450, $1370,1310,1235,1121,1065,978,884,755,699 \mathrm{~cm}^{-1} \cdot{ }^{1} \mathrm{H}$ NMR $\left(500 \mathrm{MHz}, \mathrm{CDCl}_{3}\right): \delta 6.55(\mathrm{dd}, J=14.4,6.0 \mathrm{~Hz}, 1 \mathrm{H}), 6.29(\mathrm{ddd}, J=$ $15.2,10.8,1.2 \mathrm{~Hz}, 1 \mathrm{H}), 6.19$ (dd, $J=14.4,1.3 \mathrm{~Hz}, 1 \mathrm{H}), 5.89$ (dd, $J$ $=10.7,1.5 \mathrm{~Hz}, 1 \mathrm{H}), 5.60(\mathrm{dd}, J=15.3,7.4 \mathrm{~Hz}, 1 \mathrm{H}), 4.62(\mathrm{~d}, J=$ $6.0 \mathrm{~Hz}, 1 \mathrm{H}), 3.51(\mathrm{dd}, J=9.7,6.4 \mathrm{~Hz}, 1 \mathrm{H}), 3.43(\mathrm{dd}, J=9.7$, $6.9 \mathrm{~Hz}, 1 \mathrm{H}), 3.31(\mathrm{~d}, J=9.9 \mathrm{~Hz}, 1 \mathrm{H}), 3.11(\mathrm{~s}, 3 \mathrm{H}), 2.40(\mathrm{p}, J=$ $6.7 \mathrm{~Hz}, 1 \mathrm{H}), 1.62(\mathrm{~m}, 1 \mathrm{H}), 1.59(\mathrm{~s}, 3 \mathrm{H}), 1.03(\mathrm{~d}, J=6.8 \mathrm{~Hz}, 3 \mathrm{H})$, $0.92(\mathrm{~s}, 9 \mathrm{H}), 0.89$ (s, 9H), 0.63 (d, $J=7.0 \mathrm{~Hz}, 3 \mathrm{H}), 0.06(\mathrm{~s}, 3 \mathrm{H})$, $0.04(\mathrm{~s}, 3 \mathrm{H}), 0.03(\mathrm{~s}, 3 \mathrm{H}), 0.02(\mathrm{~s}, 3 \mathrm{H}) .{ }^{13} \mathrm{C} \mathrm{NMR}(126 \mathrm{MHz}$, $\left.\mathrm{CDCl}_{3}\right): \delta 149.2,137.3,133.4,130.3,125.4,87.9,75.5,73.2,67.9$, $55.5,41.2$, 39.7, 29.7, 18.4, 18.2, 16.5, 10.5, 8.9, -4.1, -5.29, -5.32. HRMS-TOF (ESI+) calcd for $\mathrm{C}_{27} \mathrm{H}_{53} \mathrm{IO}_{3} \mathrm{Si}_{2} \mathrm{Na}^{+}(\mathrm{M}+\mathrm{Na})$ : 631.2476. Found 631.2476.

$(S, E)-2,2,3,3,7,11,11,12,12-N o n a m e t h y l-5-((S, 3 Z, 5 E)-4-$ methyl-6-(4,4,5,5-tetramethyl-1,3,2-dioxaborolan-2-yl)hepta-3,5dien-2-yl)-4,10-dioxa-3,11-disilatridec-6-ene (10). To a solution ${ }^{t} \mathrm{BuLi}\left(0.1 \mathrm{~mL}, 1.7 \mathrm{M}\right.$ solution in pentane) in $\mathrm{Et}_{2} \mathrm{O}(1.0 \mathrm{~mL})$ at $-78{ }^{\circ} \mathrm{C}$ was added iodide 9 (50 $\mathrm{mg}, 0.08 \mathrm{mmol}$ ) and the mixture was stirred for $5 \mathrm{~min}$ before adding triisopropoxyborate $(30 \mu \mathrm{L}$, $1.5 \mathrm{mmol}$ ) and the reaction was stirred for $30 \mathrm{~min}$. Pinacol (20 mg, $2.0 \mathrm{mmol}$ ) was then added and the solution was warmed to room temperature and stirred for $15 \mathrm{~h}$. The reaction was diluted with MTBE $(20 \mathrm{~mL})$ and washed with aq. $\mathrm{NH}_{4} \mathrm{Cl}(20 \mathrm{~mL})$, 
water $(20 \mathrm{~mL})$, and brine $(20 \mathrm{~mL})$. The organic phase was dried over $\mathrm{MgSO}_{4}$, filtered, and concentrated in vacuo. Purification by flash column chromatography on silica (10:1 hexanes : ethyl acetate) afforded 10 (45 mg, 93\%) as an oil.

$[\alpha]_{\mathrm{D}}^{20}=+3.2\left(c 1.0, \mathrm{CH}_{2} \mathrm{Cl}_{2}\right)$. IR (ATR) 3056, 2973, 1640, 1565, 1350, 1194, 1067, 910, $730 \mathrm{~cm}^{-1}$. ${ }^{1} \mathrm{H}$ NMR (500 $\mathrm{MHz}, \mathrm{CDCl}_{3}$ ): $\delta 6.70(\mathrm{~s}, 1 \mathrm{H}), 5.10(\mathrm{dd}, J=9.1,1.2 \mathrm{~Hz}, 1 \mathrm{H}), 5.05(\mathrm{~d}, J=9.8 \mathrm{~Hz}$, $1 \mathrm{H}), 4.16(\mathrm{dd}, J=9.0,5.5 \mathrm{~Hz}, 1 \mathrm{H}), 3.60(\mathrm{t}, J=6.2 \mathrm{~Hz}, 2 \mathrm{H}), 2.65$ (ddd, $J=9.7,6.9,5.6 \mathrm{~Hz}, 1 \mathrm{H}), 1.97$ (s, 2H), 1.88 (d, $J=1.6 \mathrm{~Hz}$, $3 \mathrm{H}), 1.85(\mathrm{~d}, J=1.4 \mathrm{~Hz}, 3 \mathrm{H}), 1.57$ (d, $J=1.4 \mathrm{~Hz}, 3 \mathrm{H}), 1.53-1.40$ $(\mathrm{m}, 4 \mathrm{H}), 1.28$ (s, 9H), 0.89 (s, 12H), 0.87 (d, J=7.0 Hz, 3H), 0.85 (s, 9H), 0.04 (s, 6H), -0.01 (s, 3H), -0.04 (s, 3H). ${ }^{13} \mathrm{C}$ NMR (125 $\left.\mathrm{MHz}, \mathrm{CDCl}_{3}\right): \delta 138.1,135.3,133.4,132.7,127.2,83.3,72.9,63.0$, 39.4, 39.4, 32.5, 26.0, 25.90, 25.86, 24.8, 24.7, 24.0, 23.9, 23.0, 18.4, 18.2, 16.7, 16.0, -4.3, -4.8, -5.3. HRMS-TOF (ESI+) calcd for $\mathrm{C}_{34} \mathrm{H}_{67} \mathrm{BO}_{4} \mathrm{Si}_{2} \mathrm{Na}^{+}(\mathrm{M}+\mathrm{Na}): 629.4569$. Found 629.4572.

$(2 S, 3 R, E)-9-(($ tert-Butyldimethylsilyl)oxy)-1-((4methoxybenzyl)oxy)-2,5-dimethylnon-4-en-3-yl

2-(diethoxyphosphoryl) propanoate (13). To a solution of alcohol 11 (500 $\mathrm{mg}, 1.2 \mathrm{mmol}$ ) and phosphate $12(249 \mathrm{mg}, 1.2 \mathrm{mmol})$ in DCM $(1.6 \mathrm{~mL})$ at $0{ }^{\circ} \mathrm{C}$ was added DCC (293 $\left.\mathrm{mg}, 1.42 \mathrm{mmol}\right)$ and the solution was allowed to warm to room temperature and stir for $24 \mathrm{~h}$. The reaction mixture was filtered through celite, diluted with $\mathrm{H}_{2} \mathrm{O}(10 \mathrm{~mL})$, and extracted with DCM $(2 \times 10 \mathrm{~mL})$. The combined organic extracts were dried over $\mathrm{MgSO}_{4}$, filtered and concentrated in vacuo. Purification by flash column chromatography on silica (4:1 to $1: 1$ to $1: 2$ hexanes : ethyl acetate) afforded ester 13 (604 $\mathrm{mg}, 82 \%)$ as an oil.

Spectral data for the mixture of diastereomers. IR (ATR) 2931, 2856, 1730, 1612, 1586, 1513, 1460, 1386, 1301, 1246, 1172, 1093, 1023, 962, 904, 834, 818, 774, 734, $661 \mathrm{~cm}^{-1} \cdot{ }^{1} \mathrm{H}$ NMR $(500$ $\mathrm{MHz}_{\mathrm{CDCl}} \mathrm{CD}: \delta 7.25-7.22(\mathrm{~m}, 4 \mathrm{H}), 6.86(\mathrm{~d}, J=8.7 \mathrm{~Hz}, 4 \mathrm{H}), 5.61$ $(\mathrm{dd}, J=9.7,6.5 \mathrm{~Hz}, 1 \mathrm{H}), 5.57(\mathrm{dd}, J=9.7,6.7 \mathrm{~Hz}, 1 \mathrm{H}), 5.09(\mathrm{~d}, J=$ $9.7 \mathrm{~Hz}, 2 \mathrm{H}), 4.41$ (d, $J=10.2 \mathrm{~Hz}, 2 \mathrm{H}), 4.37$ (d, $J=10.2 \mathrm{~Hz}, 2 \mathrm{H})$, 4.16-4.07 (m, 8H), 3.80 (s, 6H), 3.59 (t, $J=5.9 \mathrm{~Hz}, 4 \mathrm{H}), 3.42(\mathrm{dd}, J$ $=9.3,5.8 \mathrm{~Hz}, 1 \mathrm{H}), 3.35(\mathrm{dd}, J=9.3,6.1 \mathrm{~Hz}, 1 \mathrm{H}), 3.30(\mathrm{dd}, J=9.3$, $6.3 \mathrm{~Hz}, 1 \mathrm{H}), 3.31$ (dd, $J=9.3,6.1 \mathrm{~Hz}, 1 \mathrm{H}), 2.95(\mathrm{dq}, J=23.3$, $7.4 \mathrm{~Hz}, 2 \mathrm{H}), 2.16(\mathrm{~m}, 12 \mathrm{H}), 2.11(\mathrm{~m}, 2 \mathrm{H}), 2.02(\mathrm{~m}, 4 \mathrm{H}), 1.72(\mathrm{~s}$, $6 \mathrm{H}), 1.47-1.36(\mathrm{~m}, 8 \mathrm{H}), 1.33-1.27(\mathrm{~m}, 6 \mathrm{H}), 0.93(\mathrm{~d}, J=7.0 \mathrm{~Hz}$, $3 \mathrm{H}), 0.92(\mathrm{~d}, J=7.0 \mathrm{~Hz}, 3 \mathrm{H}), 0.88(\mathrm{~s}, 18 \mathrm{H}), 0.03(\mathrm{~s}, 12 \mathrm{H}) \cdot{ }^{13} \mathrm{C}$ NMR (125 MHz, $\left.\mathrm{CDCl}_{3}\right): \delta 159.1,142.3,130.6,129.2,120.4$, 113.72, 113.70, 73.7, 72.7, 71.7, 62.9, 62.5, 55.3, 40.0, 39.5, 37.8, 32.41, 32.35, 26.0, 24.0, 18.4, 16.8, 12.7, 11.8, -5.3. HRMS-TOF (ESI+) calcd for $\mathrm{C}_{32} \mathrm{H}_{57} \mathrm{O}_{8} \mathrm{PSiNa}^{+}(\mathrm{M}+\mathrm{Na})$ : 651.3458. Found 651.3458.

(2S,3R,E)-9-((tert-Butyldimethylsilyl)oxy)-1-hydroxy-2,5dimethylnon-4-en-3-yl-2-(diethoxyphosphoryl)propanoate. To a solution of $13(250 \mathrm{mg}, 0.398 \mathrm{mmol})$ in a DCM : $\mathrm{H}_{2} \mathrm{O}$ mixture (20 mL : $0.2 \mathrm{~mL})$, DDQ (180 mg, $0.795 \mathrm{mmol}$ ) was added portion wise and stirred at room temperature for $1 \mathrm{~h}$. The resulting solution was quenched with aqueous sodium bicarbonate (50 $\mathrm{mL})$ and extracted with DCM $(2 \times 50 \mathrm{~mL})$. The combined organic extracts were dried over $\mathrm{MgSO}_{4}$, filtered and concentrated in vacuo. Purification by flash column chromatography on silica (1:2 to $0: 1$ hexanes : ethyl acetate) afforded the corresponding alcohol (168 $\mathrm{mg}, 83 \%)$ as an oil.
Spectral data for the mixture of diastereomers. IR (ATR) 3406, 2928, 2856, 1471, 1455, 1384, 1360, 1253, 1100, 1081, 1030, 1002, 977, 935, 834, 773, 737, $661 \mathrm{~cm}^{-1} .{ }^{1} \mathrm{H}$ NMR $(500 \mathrm{MHz}$, $\left.\mathrm{CDCl}_{3}\right): \delta 5.43(\mathrm{dd}, J=8.7,8.7 \mathrm{~Hz}, 1 \mathrm{H}), 5.39(\mathrm{dd}, J=9.4,9.4 \mathrm{~Hz}$, $1 \mathrm{H}), 5.11$ (d, $J=9.2 \mathrm{~Hz}, 1 \mathrm{H}), 5.06$ (d, $J=9.8 \mathrm{~Hz}, 1 \mathrm{H}), 4.17-4.09$ $(\mathrm{m}, 8 \mathrm{H}), 3.75(\mathrm{dd}, J=11.5,3.3 \mathrm{~Hz}, 1 \mathrm{H}), 3.64(\mathrm{dd}, J=11.5,3.8 \mathrm{~Hz}$, $1 \mathrm{H}), 3.58(\mathrm{t}, J=6.1 \mathrm{~Hz}, 4 \mathrm{H}), 3.50(\mathrm{dd}, J=11.0,4.9 \mathrm{~Hz}, 1 \mathrm{H}), 3.48$ $(\mathrm{dd}, J=11.5,4.5 \mathrm{~Hz}, 1 \mathrm{H}), 2.99(\mathrm{dq}, J=23.0,7.5 \mathrm{~Hz}, 2 \mathrm{H}), 2.00(\mathrm{~m}$, $12 \mathrm{H}), 1.83(\mathrm{~m}, 2 \mathrm{H}), 1.73(\mathrm{~d}, J=1.2 \mathrm{~Hz}, 3 \mathrm{H}), 1.71$ (d, $J=1.2 \mathrm{~Hz}$, $3 \mathrm{H}), 1.70(\mathrm{~m}, 2 \mathrm{H}), 1.65(\mathrm{~m}, 2 \mathrm{H}), 1.48-1.41(\mathrm{~m}, 4 \mathrm{H}), 1.39$ (d, $J=$ $7.0 \mathrm{~Hz}, 3 \mathrm{H}), 1.35(\mathrm{~d}, J=7.0 \mathrm{~Hz}, 3 \mathrm{H}), 1.34-1.28(\mathrm{~m}, 4 \mathrm{H}), 0.91(\mathrm{~d}, J$ $=7.0 \mathrm{~Hz}, 3 \mathrm{H}), 0.89(\mathrm{~d}, J=7.0 \mathrm{~Hz}, 3 \mathrm{H}), 0.87(\mathrm{~s}, 18 \mathrm{H}), 0.02(\mathrm{~s}$, 12H). ${ }^{13} \mathrm{C}$ NMR (125 MHz, $\mathrm{CDCl}_{3}$ ): $\delta 169.3,141.9,122.3,75.6$, 75.1, 64.2, 62.9, 40.3, 39.4, 32.3, 26.0, 23.9, 18.3, 16.8, 16.4, 13.8, 11.7, -5.3. HRMS-TOF (ESI+) calcd for $\mathrm{C}_{24} \mathrm{H}_{49} \mathrm{O}_{7} \mathrm{PSiNa}^{+}(\mathrm{M}+$ $\mathrm{Na})$ : 531.2883. Found 531.2883.

(5S,6S)-6-((E)-6-((tert-Butyldimethylsilyl)oxy)-2-methylhex-1en-1-yl)-3,5-dimethyl-5,6-dihydro-2H-pyran-2-one (14). To a solution of the alcohol (624 mg, $1.23 \mathrm{mmol})$ in DCM $(6.13 \mathrm{~mL})$ at $0{ }^{\circ} \mathrm{C}$ was added $\mathrm{NaHCO}_{3}(515 \mathrm{mg}, 6.13 \mathrm{mmol})$ followed by the portionwise addition of Dess-Martin periodinane $(520 \mathrm{mg}, 1.23$ $\mathrm{mmol}$ ) and the resulting mixture was stirred for $1 \mathrm{~h}$. The solution then was diluted with DCM $(20 \mathrm{~mL})$ and stirred vigorously with sodium thiosulfate pentahydrate $(50 \mathrm{~mL})$ for $30 \mathrm{~min}$. The layers were separated and the aqueous phase was extracted with DCM $(2 \times 50 \mathrm{~mL})$. The combined organic extracts were dried over $\mathrm{MgSO}_{4}$, filtered and concentrated in vacuo. The crude aldehyde (371 mg, 60\%) was used directly in the next reaction.

$\mathrm{Ba}(\mathrm{OH})_{2} \cdot 8 \mathrm{H}_{2} \mathrm{O}(340 \mathrm{mg}, 1.1 \mathrm{mmol})$ was heated at $120{ }^{\circ} \mathrm{C}$ for 1.5 hours under vacuum and then allowed to cool to room temperature. THF ( $7 \mathrm{~mL}$ ) was added to the flask and the solution was stirred for 10 minutes before adding the aldehyde (181 $\mathrm{mg}, 0.36 \mathrm{mmol}$ ) and the resulting mixture was stirred at room temperature for $15 \mathrm{~h}$. The reaction was quenched with aq. $\mathrm{NaHCO}_{3}(20 \mathrm{~mL})$ and extracted with MTBE $(2 \times 20 \mathrm{~mL})$. The combined organic extracts were dried over $\mathrm{MgSO}_{4}$, filtered and concentrated in vacuo. Purification by flash column chromatography on silica (20:1 to $10: 1$ to $4: 1$ hexanes : ethyl acetate) afforded lactone $14(72.2 \mathrm{mg}, 57 \%)$ as an oil.

$[\alpha]_{\mathrm{D}}^{20}=-2.8\left(c 1.0, \mathrm{CH}_{2} \mathrm{Cl}_{2}\right)$. IR (ATR) 2952, 2928, 2856, 1716, 1460, 1359, 1253, 1226, 1199, 1154, 1134, 1099, 1005, 981, 834, 810, 773, 731, 661. ${ }^{1} \mathrm{H}$ NMR (500 MHz, $\left.\mathrm{CDCl}_{3}\right): \delta 6.34(\mathrm{~s}, 1 \mathrm{H})$, $5.22(\mathrm{~d}, J=9.0 \mathrm{~Hz}, 1 \mathrm{H}), 4.68(\mathrm{dd}, J=10.5,9.0 \mathrm{~Hz}, 1 \mathrm{H}), 3.57(\mathrm{~m}$, $2 \mathrm{H}$ ), 2.45 (ddtt, $J=14.6,7.2,4.8,2.4 \mathrm{~Hz}, 1 \mathrm{H}), 2.03(\mathrm{t}, J=5.4 \mathrm{~Hz}$, $2 \mathrm{H}), 1.87$ (dd, $J=2.4,1.5 \mathrm{~Hz}, 3 \mathrm{H}), 1.67$ (d, $J=1.5 \mathrm{~Hz}, 3 \mathrm{H}), 1.50-$ $1.41(\mathrm{~m}, 3 \mathrm{H}), 0.98$ (d, $J=7.3 \mathrm{~Hz}, 3 \mathrm{H}), 0.85$ (d, $J=6.0 \mathrm{~Hz}, 1 \mathrm{H})$, 0.85 (s, 9H), 0.00 (s, 6H). ${ }^{13} \mathrm{C}$ NMR (125 MHz, $\left.\mathrm{CDCl}_{3}\right): \delta 165.96$, 145.52, 143.82, 127.37, 121.50, 80.36, 62.86, 39.24, 34.59, 32.30, 25.95, 23.78, 18.31, 16.88, 15.94, -5.30. HRMS-TOF (ESI+) calcd for $\mathrm{C}_{20} \mathrm{H}_{36} \mathrm{O}_{3} \mathrm{SiH}^{+}(\mathrm{M}+\mathrm{H})$ : 353.2512. Found 353.2516.

$(4 Z, 6 S, 7 S, 8 E)-13-(($ tert-Butyldimethylsilyl)oxy)-4,6,9trimethyl-2-(4,4,5,5-tetramethyl-1,3,2-dioxaborolan-2-yl)trideca2,4,8-trien-7-ol (15). To a solution of lactone $14(175 \mathrm{mg}, 0.498$ $\mathrm{mmol})$ in DCM $(5 \mathrm{~mL})$ at $-78{ }^{\circ} \mathrm{C}$ was added DIBAL-H $(0.106 \mathrm{~mL}$, $0.597 \mathrm{mmol}$ ) dropwise over $10 \mathrm{~min}$ and the resulting mixture was stirred for 1 hour. The reaction was quenched with the 
addition of aq. Rochelle's salt $(2.0 \mathrm{M}, 20 \mathrm{~mL})$ and the solution was stirred vigorously for $1 \mathrm{~h}$. The layers were separated and the aqueous phase was extracted with DCM $(2 \times 20 \mathrm{~mL})$. The combined organic extracts were dried over $\mathrm{MgSO}_{4}$, filtered and concentrated in vacuo. The resulting lactol product $(141 \mathrm{mg}$, $80 \%)$ could be used without further purification.

${ }^{1} \mathrm{H}$ NMR (500 MHz, $\left.\mathrm{CDCl}_{3}\right): \delta 5.48(\mathrm{~s}, 1 \mathrm{H}), 5.16(\mathrm{~m}, 2 \mathrm{H}), 4.24$ $(\mathrm{t}, J=9.3 \mathrm{~Hz}, 1 \mathrm{H}), 3.61(\mathrm{t}, J=6.0 \mathrm{~Hz}, 3 \mathrm{H}), 2.89(\mathrm{~s}, 1 \mathrm{H}), 2.09(\mathrm{~m}$, $1 \mathrm{H}), 2.06(\mathrm{~m}, 2 \mathrm{H}), 1.73(\mathrm{~m}, 3 \mathrm{H}), 1.71(\mathrm{~d}, J=1.5 \mathrm{~Hz}, 3 \mathrm{H}), 1.50(\mathrm{~m}$, $3 \mathrm{H}), 0.89$ (s, 9H), 0.86 (d, $J=7.1 \mathrm{~Hz}, 3 \mathrm{H}), 0.04$ (s, 6H). ${ }^{13} \mathrm{C} \mathrm{NMR}$ $\left(125 \mathrm{MHz}, \mathrm{CDCl}_{3}\right): \delta 141.98,132.03,129.75,12.65,91.94,69.63$, $63.01,39.38,35.14,32.41,25.97,23.96,18.93,18.35,16.95$, $16.38,-5.27$.

To a solution of tetramethylpiperidine $(137 \mathrm{mg}, 0.97 \mathrm{mmol})$ in THF $(0.5 \mathrm{~mL})$ at $-78{ }^{\circ} \mathrm{C}$ was added $n$-BuLi $(0.388 \mathrm{~mL}, 2.5 \mathrm{M})$. The solution was allowed to warm to $0{ }^{\circ} \mathrm{C}$ before adding 1,1 bis(pinacolboronato)ethane $(187 \mathrm{mg}, 0.65 \mathrm{mmol})$. After 10 minutes, the lactol $(114.6 \mathrm{mg}, 0.32 \mathrm{mmol}$ ) was added and the solution was allowed to slowly warm to room temperature and stirred for $15 \mathrm{~h}$. The solution was diluted with diethyl ether (10 $\mathrm{mL}$ ), filtered through a silica plug, and concentrated in vacuo. Purification by flash column chromatography on silica $(20: 1$ to $10: 1$ to $4: 1$ hexanes : ethyl acetate) afforded the vinyl boronate 15 (90 $\mathrm{mg}, 57 \%$ ) as a $3: 1$ mixture of $Z: E$ isomers.

Spectral data for Z-15. IR (ATR) 2977, 2933, 2879, 1460, 1305, 1268, 1215, 1142, 1105, 1016, 967, 846, 775, 736, 669. ${ }^{1} \mathrm{H}$ NMR $\left(500 \mathrm{MHz}, \mathrm{CDCl}_{3}\right): \delta 6.74(\mathrm{~s}, 1 \mathrm{H}), 5.17-5.09(\mathrm{~m}, 2 \mathrm{H}), 4.04(\mathrm{dd}, J=$ 8.9, $7.5 \mathrm{~Hz}, 1 \mathrm{H}), 3.61(\mathrm{t}, J=5.9 \mathrm{~Hz}, 2 \mathrm{H}), 2.34(\mathrm{~m}, 1 \mathrm{H}), 2.03(\mathrm{~m}$, $2 \mathrm{H}), 1.84(\mathrm{~s}, 3 \mathrm{H}), 1.71(\mathrm{~s}, 3 \mathrm{H}), 1.65(\mathrm{~s}, 3 \mathrm{H}) 1.53-1.43(\mathrm{~m}, 4 \mathrm{H}), 1.29$ $(\mathrm{s}, 12 \mathrm{H}), 1.22(\mathrm{~d}, J=3.8 \mathrm{~Hz}, 3 \mathrm{H}), 0.89(\mathrm{~s}, 9 \mathrm{H}), 0.04(\mathrm{~s}, 6 \mathrm{H}) \cdot{ }^{13} \mathrm{C}$ NMR $\left(125 \mathrm{MHz}, \mathrm{CDCl}_{3}\right): \delta 142.5,139.7,136.5,130.3,125.7,83.1$, 72.2, 63.1, 39.5, 32.5, 29.7, 26.0, 24.8, 24.0, 23.7, 18.4, 16.7, 16.2, 15.4, 10.4, -5.3. HRMS-TOF (ESI+) calcd for $\mathrm{C}_{28} \mathrm{H}_{53} \mathrm{BO}_{4} \mathrm{SiNa}^{+}(\mathrm{M}$ $+\mathrm{Na})$ : 515.3704. Found 515.3706.

Spectral data for $\boldsymbol{E}-15 .{ }^{1} \mathrm{H}$ NMR $\left(500 \mathrm{MHz}, \mathrm{CDCl}_{3}\right): \delta 6.68(\mathrm{~s}$, $1 \mathrm{H}), 5.13(\mathrm{~d}, 9.24 \mathrm{~Hz}, 1 \mathrm{H}) 5.07$ (d, $J=9.24 \mathrm{~Hz}, 1 \mathrm{H}), 4.01(\mathrm{t}, J=$ $8.46 \mathrm{~Hz}, 1 \mathrm{H}), 3.60(\mathrm{~m}, 2 \mathrm{H}), 2.50(\mathrm{~m}, 1 \mathrm{H}), 2.04(\mathrm{~d}, J=5.90 \mathrm{~Hz}$, $2 \mathrm{H}), 1.88(\mathrm{dd}, J=5.34,1.34 \mathrm{~Hz}, 4 \mathrm{H}) 1.66(\mathrm{~s}, 3 \mathrm{H}), 1.48(\mathrm{~m}, 1 \mathrm{H})$ $1.24(\mathrm{~d}, J=3.31 \mathrm{~Hz}, 3 \mathrm{H}), 1.21(\mathrm{~d}, 4.15 \mathrm{~Hz}, 12 \mathrm{H}), 1.04(\mathrm{~d}, J=$ $7.31 \mathrm{~Hz}, 2 \mathrm{H}), 0.89(\mathrm{~s}, 9 \mathrm{H}), 0.84(\mathrm{~d}, J=6.73 \mathrm{~Hz}, 2 \mathrm{H}), 0.72(\mathrm{q}, J=$ $7.29 \mathrm{~Hz}, 1 \mathrm{H}) 0.04(\mathrm{~s}, 6 \mathrm{H}) .{ }^{13} \mathrm{C} \mathrm{NMR}\left(125 \mathrm{MHz}, \mathrm{CDCl}_{3}\right): \delta 140.12$, 139.32, 136.49, 130.68, 126.10, 82.85, 72.41, 63.01, 40.19, 39.50, $32.45,25.94,24.81,24.51,24.08,23.94,23.23,18.31,16.80,9.03$, -5.30 .

Compound 1. To a degassed solution of vinyl boronate 9 (60 mg, $0.1 \mathrm{mmol}$ ) and vinyl iodide 2 (61 $\mathrm{mg}, 0.1 \mathrm{mmol}$ ) in DMF $(1 \mathrm{~mL})$ were added $\mathrm{Pd}(\mathrm{dppf}) \mathrm{Cl}_{2}(4 \mathrm{mg}, 0.005 \mathrm{mmol})$ and $\mathrm{Cs}_{2} \mathrm{CO}_{3}$ (40 $\mathrm{mg}, 0.12 \mathrm{mmol}$ ) and the resulting solution was stirred at room temperature for $8 \mathrm{~h}$. The reaction was diluted with MTBE $(20 \mathrm{~mL})$ and washed with water $(20 \mathrm{~mL})$. The layers were separated and the aqueous phase was extracted with MTBE $(20 \mathrm{~mL})$. The combined organic extracts were dried over $\mathrm{MgSO}_{4}$ and concentrated in vacuo. Purification by flash column chromatography on silica (20:1 to $10: 1$ hexanes : ethyl acetate) afforded the coupled product $\mathbf{1}(77 \mathrm{mg}, 80 \%)$ as an oil.

$[\alpha]_{\mathrm{D}}^{20}=-5.2\left(c 0.5, \mathrm{CH}_{2} \mathrm{Cl}_{2}\right)$. IR (ATR) 3062, 2983, 1736, 1614, 1415, 1274, 1267, 1129, 1078, $930 \mathrm{~cm}^{-1}$. ${ }^{1} \mathrm{H}$ NMR $(500 \mathrm{MHz}$,
$\left.\mathrm{C}_{6} \mathrm{D}_{6}\right): \delta 6.80(\mathrm{~d}, J=16.0 \mathrm{~Hz}, 1 \mathrm{H}), 6.44(\mathrm{ddd}, J=15.3,10.7$, $0.9 \mathrm{~Hz}, 1 \mathrm{H}), 6.17$ (d, $J=10.4 \mathrm{~Hz}, 1 \mathrm{H}), 6.02(\mathrm{~s}, 1 \mathrm{H}), 5.90$ (dd, $J=$ $16.0,7.2 \mathrm{~Hz}, 1 \mathrm{H}$ ), 5.58 (dd, $J=15.4,8.0 \mathrm{~Hz}, 1 \mathrm{H}), 5.34(\mathrm{dd}, J=8.4$, $0.8 \mathrm{~Hz}, 1 \mathrm{H}), 5.29$ (ddd, $J=9.8,2.0,1.0 \mathrm{~Hz}, 1 \mathrm{H}), 5.07$ (d, $J=$ $7.4 \mathrm{~Hz}, 1 \mathrm{H}), 4.29$ (dd, $J=9.0,6.0 \mathrm{~Hz}, 1 \mathrm{H}), 3.63(\mathrm{~d}, J=10.0 \mathrm{~Hz}$, $1 \mathrm{H}), 3.58-3.53(\mathrm{~m}, 2 \mathrm{H}), 3.47$ (dd, $J=9.7,6.2 \mathrm{~Hz}, 1 \mathrm{H}), 3.38$ (dd, $J$ $=9.7,6.8 \mathrm{~Hz}, 1 \mathrm{H}), 3.18(\mathrm{~s}, 3 \mathrm{H}), 2.71(\mathrm{~m}, 1 \mathrm{H}), 2.42(\mathrm{~m}, 1 \mathrm{H}), 1.96$ $(\mathrm{m}, 2 \mathrm{H}), 1.91(\mathrm{~s}, 3 \mathrm{H}), 1.86(\mathrm{~m}, 1 \mathrm{H}), 1.89(\mathrm{~d}, J=1.0 \mathrm{~Hz}, 3 \mathrm{H}), 1.71$ (d, $J=1.0 \mathrm{~Hz}, 3 \mathrm{H}), 1.59$ (s, 3H), 1.50 (m, 2H), 1.39 (m, 2H), 1.08 $(\mathrm{s}, 6 \mathrm{H}), 1.03(\mathrm{~s}, 6 \mathrm{H}), 1.02(\mathrm{~s}, 3 \mathrm{H}), 1.01(\mathrm{~s}, 6 \mathrm{H}), 1.00(\mathrm{~s}, 3 \mathrm{H}), 0.99$ $(\mathrm{d}, J=7.0 \mathrm{~Hz}, 3 \mathrm{H}), 0.98(\mathrm{~s}, 3 \mathrm{H}), 0.96(\mathrm{~d}, J=7.0 \mathrm{~Hz}, 3 \mathrm{H}), 0.95$ (s, $6 \mathrm{H}), 0.94(\mathrm{~d}, J=7.0 \mathrm{~Hz}, 3 \mathrm{H}), 0.93(\mathrm{~s}, 3 \mathrm{H}), 0.19(\mathrm{~s}, 3 \mathrm{H}), 0.17$ (s, $3 \mathrm{H}), 0.13(\mathrm{~s}, 6 \mathrm{H}), 0.08$ (s, 6H) $0.05(\mathrm{~s}, 6 \mathrm{H}) .{ }^{13} \mathrm{C} \mathrm{NMR}(125 \mathrm{MHz}$, $\left.\mathrm{C}_{6} \mathrm{D}_{6}\right): \delta{ }^{13} \mathrm{C}$ NMR (126 MHz, $\left.\mathrm{C}_{6} \mathrm{D} 6\right) \delta 137.8,136.0,134.9,134.7$, 133.4, 133.3, 133.0, 132.5, 130.9, 130.3, 126.3, 125.4, 73.8, 72.9, 68.5, 63.4, 63.4, 56.0, 43.7, 41.5, 40.6, 40.1, 33.1, 31.4, 30.3, 28.5, 26.7, 26.6, 26.5, 25.4, 24.8, 24.8, 24.0, 21.0, 19.1, 18.9, 18.8, 17.4, 17.2, 17.1, 16.2, 14.3, 11.2, 9.8, 8.7, -3.0, -3.6, -4.2, -4.3, -4.4, $-4.7,-4.8,-4.9$. HRMS (ESI+): calcd for $\mathrm{C}_{55} \mathrm{H}_{108} \mathrm{O}_{5} \mathrm{Si}_{4} \mathrm{Na}^{+}(\mathrm{M}+$ $\mathrm{Na})^{+}: 983.7172$. Found 983.7179 .

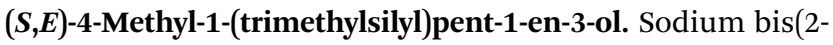
methoxyethoxy)aluminum hydride $(2.65 \mathrm{~mL}, 3.07 \mathrm{M})$ was added dropwise to a solution of $(S)-19^{26}(1.0313 \mathrm{~g}, 6.05 \mathrm{mmol})$ in diethyl ether $(15 \mathrm{~mL})$ at $0{ }^{\circ} \mathrm{C}$ and the mixture was stirred to room temperature for 15 hours. The reaction was quenched with ethyl acetate $(50 \mathrm{~mL})$ and stirred vigorously with aq. Rochelle's salt $(50 \mathrm{~mL}, 2.0 \mathrm{M})$ for 1 hour. The layers were separated and the aqueous phase was extracted with ethyl acetate $(2 \times 50 \mathrm{~mL})$. The combined organic fractions were dried over $\mathrm{MgSO}_{4}$, filtered through cotton, and concentrated under vacuum. Purification by flash chromatography on silica (20:1 to 10:1 hexanes : ethyl acetate) gave the trans-alkene product $(0.859 \mathrm{~g}$, $4.98 \mathrm{mmol}, 82.4 \%)$ as an oil.

$[\alpha]_{\mathrm{D}}^{20}=+20.2\left(c 1.0, \mathrm{CH}_{2} \mathrm{Cl}_{2}\right)$. IR (ATR): 3355, 3057, 3025, 2956, 2923, 1721, 1621, 1601, 1582, 1493, 1452, 1247, 1025, 989, 866, 835, 762, 742, 694. ${ }^{1} \mathrm{H}$ NMR (500 $\left.\mathrm{MHz}, \mathrm{CDCl}_{3}\right): \delta 6.06(\mathrm{dd}, J=$ 9.5, $5.6 \mathrm{~Hz}, 1 \mathrm{H}), 5.88$ (dd, $J=9.4,1.3 \mathrm{~Hz}, 1 \mathrm{H}), 3.88$ (s, 1H), 1.76 (o, $J=6.9 \mathrm{~Hz}, 1 \mathrm{H}), 0.94(\mathrm{~d}, J=7.1 \mathrm{~Hz}, 3 \mathrm{H}), 0.92(\mathrm{~d}, J=7.2 \mathrm{~Hz}$, $3 \mathrm{H}), 0.10$ (s, 9H). ${ }^{13} \mathrm{C} \mathrm{NMR}\left(500 \mathrm{MHz}, \mathrm{CDCl}_{3}\right): \delta$ 146.98, 130.48, 79.61, 33.55, 18.35, 17.64, -1.26. HRMS (ESI+): calcd for $\mathrm{C}_{9}$ $\mathrm{H}_{20} \mathrm{OSiNa}^{+}(\mathrm{M}+\mathrm{Na})^{+}:$195.1181. Found 195.1180.

(S,E)-Triethyl((4-methyl-1-(trimethylsilyl)pent-1-en-3-yl)oxy) silane. Chlorotriethylsilane $(0.117 \mathrm{~mL}, 0.696 \mathrm{mmol})$ was added to a solution of the alcohol $(0.100 \mathrm{~g}, 0.580 \mathrm{mmol})$ and imidazole $(0.0790 \mathrm{~g}, 1.16 \mathrm{mmol})$ in $\mathrm{DCM}(1.66 \mathrm{~mL})$ at $0{ }^{\circ} \mathrm{C}$ and the resulting mixture was warmed to room temperature and stirred for 3 hours. The reaction was quenched with water $(10 \mathrm{~mL})$ and extracted with DCM $(2 \times 10 \mathrm{~mL})$. The combined organic extracts were dried over $\mathrm{MgSO}_{4}$, filtered through cotton, and concentrated under vacuum. Purification by flash chromatography on silica (20:1 hexanes : ethyl acetate) gave the TES-protected alcohol $(0.1453 \mathrm{~g}, 0.501 \mathrm{mmol}, 86.3 \%)$ as an oil.

$[\alpha]_{\mathrm{D}}^{20}=+18.0\left(c 1.0, \mathrm{CH}_{2} \mathrm{Cl}_{2}\right)$. IR (ATR): 2954, 2911, 2876, 1620, 1459, 1414, 1259, 1059, 1004, 992, 872, 834, 721, 691. ${ }^{1} \mathrm{H}$ NMR $\left(500 \mathrm{MHz}, \mathrm{CDCl}_{3}\right): \delta 5.97(\mathrm{dd}, J=18.7,6.5 \mathrm{~Hz}, 1 \mathrm{H}), 5.75(\mathrm{dd}, J=$ 18.8, 1.1 Hz, 1H), 3.77 (ddd, $J=6.4,1.0 \mathrm{~Hz}, 1 \mathrm{H}), 1.66$ (o, $J=$ $6.5 \mathrm{~Hz}, 1 \mathrm{H}), 0.96$ (t, $J=8.0 \mathrm{~Hz}, 9 \mathrm{H}), 0.90(\mathrm{~d}, J=6.8,3 \mathrm{H}), 0.85$ (d, $J$ 
$=6.8 \mathrm{~Hz}, 3 \mathrm{H}), 0.59(\mathrm{dq}, J=7.7,2.5 \mathrm{~Hz}, 6 \mathrm{H}), 0.08(\mathrm{~s}, 9 \mathrm{H}) .{ }^{13} \mathrm{C}$ NMR (500 MHz, $\left.\mathrm{CDCl}_{3}\right): \delta$ 147.91, 129.98, 81.17, 34.35, 18.39, 18.18, 6.88, 5.00, -1.32. HRMS (ESI+): calcd for $\mathrm{C}_{15} \mathrm{H}_{34} \mathrm{OSi}_{2} \mathrm{Na}^{+}$ $(\mathrm{M}+\mathrm{Na})^{+}:$309.2046. Found 309.2045.

(S,E)-Triethyl((1-iodo-4-methylpent-1-en-3-yl)oxy)silane (18). $N$-Iodosuccinimide $(0.169 \mathrm{~g}, 0.751 \mathrm{mmol})$ was added to the vinyl silane $(0.144 \mathrm{~g}, 0.501 \mathrm{mmol})$ and 2,6-lutidine $(0.081 \mathrm{~mL}, 0.701$ $\mathrm{mmol})$ in 1,1,1,3,3,3-hexafluoroisopropanol $(2.00 \mathrm{~mL})$ at $0{ }^{\circ} \mathrm{C}$ and the resulting mixture was stirred for 15 minutes. The reaction was quenched with water $(5 \mathrm{~mL})$ and extracted with DCM $(2 \times 10 \mathrm{~mL})$. The combined organic extracts were washed with sodium thiosulfate $(25 \mathrm{~mL})$, hydrochloric acid $(25 \mathrm{~mL}, 1$ $\mathrm{M})$, water $(25 \mathrm{~mL})$, and sodium bicarbonate $(25 \mathrm{~mL})$, then dried over $\mathrm{MgSO}_{4}$, filtered through cotton, and concentrated under vacuum. Purification by flash chromatography on silica $20: 1$ hexanes : ethyl acetate) gave $18(0.0885 \mathrm{~g}, 0.260 \mathrm{mmol}, 52.0 \%)$ as an oil.

$[\alpha]_{\mathrm{D}}^{20}=+8.2\left(c 0.5, \mathrm{CH}_{2} \mathrm{Cl}_{2}\right)$. IR (ATR): 2955, 2910, 2875, 1607, 1459, 1414, 1384, 1364, 1238, 1182, 1162, 1118, 1103, 1071, 1004, 948, 828, 723. ${ }^{1} \mathrm{H}$ NMR $\left(500 \mathrm{MHz}, \mathrm{CDCl}_{3}\right): \delta 6.53(\mathrm{dd}, J=$ $14.4,6.8 \mathrm{~Hz}, 1 \mathrm{H}), 6.20(\mathrm{dd}, J=14.4,1.1 \mathrm{~Hz}, 1 \mathrm{H}), 3.83$ (ddd, $J=$ 6.7, 5.7, $1.1 \mathrm{~Hz}), 1.69$ (do, $J=6.8,1.1 \mathrm{~Hz}, 1 \mathrm{H}), 0.97(\mathrm{t}, J=8.0 \mathrm{~Hz}$, $9 \mathrm{H}), 0.91(\mathrm{~d}, J=6.8,3 \mathrm{H}), 0.87(\mathrm{~d}, J=6.8,3 \mathrm{H}), 0.60(\mathrm{q}, J=8.2 \mathrm{~Hz}$, $6 \mathrm{H}) .{ }^{13} \mathrm{C}$ NMR $\left(500 \mathrm{MHz}, \mathrm{CDCl}_{3}\right.$ ): 147.81, 80.18, 76.26, 34.34, 18.01, 17.82, 6.82, 4.90. HRMS (ESI+): calcd for $\mathrm{C}_{12} \mathrm{H}_{25} \mathrm{IOSiNa}^{+}$ $(\mathrm{M}+\mathrm{Na})^{+}:$363.0617. Found 363.0612.

$(5 S, 6 E, 8 Z, 10 Z, 12 S, 13 S, 14 E)-13-(($ tert-Butyldimethylsilyl)oxy)3,3-diethyl-5-isopropyl-8,10,12,15,21,21,22,22-octamethyl-4,20dioxa-3,21-disilatricosa-6,8,10,14-tetraene (17). To a solution of vinyl boronate $\boldsymbol{E}-\mathbf{1 5}(100 \mathrm{mg}, 0.2 \mathrm{mmol})$ and vinyl iodide 18 (69 $\mathrm{mg}, 0.2 \mathrm{mmol})$ in degassed DMF (1 mL) was added Pd(dppf) $\mathrm{Cl}_{2}$ (4 mg, $0.004 \mathrm{mmol}$ ) and $\mathrm{Cs}_{2} \mathrm{CO}_{3}(40 \mathrm{mg}, 0.12 \mathrm{mmol}$ ) and the resulting solution was stirred for $15 \mathrm{~h}$. The reaction mixture was diluted with MTBE $(5 \mathrm{~mL})$ and washed with water $(20 \mathrm{~mL})$. The aqueous phase was extracted with $\operatorname{MTBE}(2 \times 20 \mathrm{~mL})$. The combined organic extracts were dried with $\mathrm{MgSO}_{4}$ and concentrated in vacuo. Purification by flash column chromatography on silica (10:1 to $4: 1$ hexanes : ethyl acetate) afforded 17 (93 mg, 80\%) as an oil. The product was unable to be purified away from a side product and this mixture was taken direction into the next reaction.

$[\alpha]_{\mathrm{D}}^{20}=-3.2\left(c 0.5, \mathrm{CH}_{2} \mathrm{Cl}_{2}\right)$. IR (ATR) 2955, 2930, 2875, 1460, 1352, 1344, 1254, 1143, 1103, 1004, 969, 835, 774, 741, 724, 666. ${ }^{1} \mathrm{H}$ NMR (500 MHz, $\left.\mathrm{CDCl}_{3}\right): \delta 6.37(\mathrm{~d}, J=15.7 \mathrm{~Hz}, 1 \mathrm{H}), 5.84(\mathrm{~s}$, $1 \mathrm{H}), 5.64(\mathrm{dd}, J=15.7,7.9 \mathrm{~Hz}, 1 \mathrm{H}), 5.16(\mathrm{~d}, 9.8 \mathrm{~Hz}, 1 \mathrm{H}), 5.10(\mathrm{~d}, J$ $=8.8 \mathrm{~Hz}, 1 \mathrm{H}), 4.02(\mathrm{t}, J=8.8 \mathrm{~Hz}, 1 \mathrm{H}), 3.82(\mathrm{t}, J=7.0 \mathrm{~Hz}, 1 \mathrm{H})$, $3.60(\mathrm{t}, J=5.9 \mathrm{~Hz}, 2 \mathrm{H}), 2.34(\mathrm{~m}, 1 \mathrm{H}), 2.02(\mathrm{~m}, 2 \mathrm{H}), 1.86(\mathrm{~s}, 3 \mathrm{H})$, $1.84(\mathrm{~s}, 3 \mathrm{H}), 1.67(\mathrm{~m}, 1 \mathrm{H}), 1.66(\mathrm{~s}, 3 \mathrm{H}), 1.53-1.43(\mathrm{~m}, 4 \mathrm{H}), 1.24$ (d, $J=3.8 \mathrm{~Hz}, 3 \mathrm{H}), 0.93(\mathrm{t}, J=7.9 \mathrm{~Hz}, 9 \mathrm{H}), 0.89(\mathrm{~s}, 9 \mathrm{H}), 0.84(\mathrm{~d}, J=$ $5.8 \mathrm{~Hz}, 3 \mathrm{H}), 0.82(\mathrm{~d}, J=5.8 \mathrm{~Hz}, 3 \mathrm{H}), 0.56(\mathrm{q}, J=7.9 \mathrm{~Hz}, 6 \mathrm{H}) 0.04$ $(\mathrm{s}, 6 \mathrm{H}) .{ }^{13} \mathrm{C}$ NMR $\left(125 \mathrm{MHz}, \mathrm{CDCl}_{3}\right): \delta 139.8,135.1,132.4,130.9$, 129.0, 128.7, 125.7, 82.9, 72.3, 63.0, 40.5, 39.5, 35.1, 32.5, 26.0, 24.8, 24.6, 20.4 18.4 16.7, 16.4, 9.1, 6.9, 5.0, -5.3. HRMS (ESI+): calcd for $\mathrm{C}_{34} \mathrm{H}_{66} \mathrm{O}_{3} \mathrm{Si}_{2} \mathrm{Na}^{+}(\mathrm{M}+\mathrm{Na})^{+}:$601.4448. Found 601.4446.

$(5 E, 7 S, 8 S, 9 Z, 11 Z, 13 E, 15 S)-5,8,10,12,16-$

Pentamethylheptadeca-5,9,11,13-tetraene-1,7,15-triol (20). To a solution of 17 (77 mg, $0.053 \mathrm{mmol})$ in THF $(1.2 \mathrm{~mL})$ and pyridine $(0.3 \mathrm{~mL})$ at $4{ }^{\circ} \mathrm{C}$ was added $\mathrm{HF}$ pyr $(0.2 \mathrm{~mL}, 60 \% \mathrm{HF})$. The reaction mixture was kept at $4{ }^{\circ} \mathrm{C}$ for $15 \mathrm{~h}$, and then quenched with aq. $\mathrm{NaHCO}_{3}(20 \mathrm{~mL})$ and extracted with ethyl acetate $(2 \times 10 \mathrm{~mL})$. The organic layers were dried over $\mathrm{MgSO}_{4}$ and concentrated in vacuo. Purification by flash column chromatography on silica (1:1 to $1: 2$ hexanes : ethyl acetate) afforded $20(30.1 \mathrm{mg}, 65 \%)$ as an oil.

$[\alpha]_{\mathrm{D}}^{20}=-2.8\left(c 0.5, \mathrm{CH}_{2} \mathrm{Cl}_{2}\right)$. IR (ATR) 3338, 2957, 2929, 2870, $1667,1638,1585,1447,1378,1366,1328,1257,1203,1175$, 1136, 1106, 1065, 1003, 973, 935, 858, 817, 769, 736, 697, 667. ${ }^{1} \mathrm{H}$ NMR (500 MHz, $\left.\mathrm{C}_{6} \mathrm{D}_{6}\right): \delta 6.84(\mathrm{~d}, J=15.8,1 \mathrm{H}), 5.78(\mathrm{dd}, J=15.8$, $6.6 \mathrm{~Hz}, 1 \mathrm{H}), 5.73(\mathrm{~s}, 1 \mathrm{H}), 5.27-5.24(\mathrm{~m}, 2 \mathrm{H}), 4.12(\mathrm{t}, J=8.0 \mathrm{~Hz}$, $1 \mathrm{H}), 3.90(\mathrm{t}, J=6.2 \mathrm{~Hz}, 1 \mathrm{H}), 3.40(\mathrm{~m}, 2 \mathrm{H}), 2.60(\mathrm{~m}, 1 \mathrm{H}), 1.89(\mathrm{~m}$, $2 \mathrm{H}), 1.81(\mathrm{~s}, 3 \mathrm{H}), 1.78(\mathrm{~s}, 3 \mathrm{H}), 1.57(\mathrm{~s}, 3 \mathrm{H}), 1.40-1.34(\mathrm{~m}, 5 \mathrm{H})$, $1.03(\mathrm{~d}, J=6.2 \mathrm{~Hz}, 3 \mathrm{H}) 0.95(\mathrm{dd}, J=11.6,7.1 \mathrm{~Hz}, 6 \mathrm{H}) .{ }^{13} \mathrm{C} \mathrm{NMR}$ (125 MHz, $\mathrm{C}_{6} \mathrm{D}_{6}$ ): $\delta 138.5,133.6,132.8,132.0,130.5,129.1,77.7$, 72.5, 62.1, 40.4, 39.1, 34.1, 32.1, 24.4, 23.7, 19.9, 18.5, 18.1, 16.8, 16.4. HRMS (ESI+): calcd for $\mathrm{C}_{22} \mathrm{H}_{38} \mathrm{O}_{3} \mathrm{Na}^{+}(\mathrm{M}+\mathrm{Na})^{+}: 373.2719$. Found 373.2711.

\section{Conflicts of interest}

There are no conflicts to declare.

\section{Acknowledgements}

Financial support from the Camille \& Henry Dreyfus Foundation is gratefully acknowledged.

\section{Notes and references}

1 G. W. O'Neil, A. M. Craig, J. R. Williams, J. C. Young and P. C. Spiegel, Synlett, 2017, 28, 1101.

2 K. von Schwarzenberg, T. Lajtos, L. Simon, R. Mueller, G. Vereb and A. M. Vollmar, Mol. Oncol., 2014, 8, 9.

3 R. Hamm, M. Zeino, S. Frewert and T. Efferth, Toxicol. Appl. Pharmacol., 2014, 281, 78.

4 S. Zhang, L. S. Schneider, B. Vick, M. Grunert, I. Jeremias, D. Menche, R. Müller, A. M. Vollmar and J. Liebl, OncoTargets Ther., 2015, 6, 43508.

5 L. S. Schneider, K. von Schwarzenberg, T. Lehr, M. Ulrich, R. Kubisch-Dohmen, J. Liebl, D. Trauner, D. Menche and A. M. Vollmar, Cancer Res., 2015, 75, 2863.

6 O. Scherer, H. Steinmetz, C. Kaether, C. Weinigel, D. Barz, H. Kleinert, D. Menche, R. Müller, C. Pergola and O. Werz, Biochem. Pharmacol., 2014, 91, 490.

7 L. S. Schneider, M. Ulrich, T. Lehr, D. Menche, R. Müller and K. von Schwarzenberg, Mol. Oncol., 2016, 10, 1054.

8 L. Thomas, Z. Rao, J. Gerstmeier, M. Raasch, C. Weinigel, S. Rummler, D. Menche, R. Müller, C. Pergola, A. Mosig and O. Werz, Biochem. Pharmacol., 2017, 130, 71.

9 K. Bartel, M. Winzi, M. Ulrich, A. Koeberle, D. Menche, O. Werz, R. Müller, J. Guck, A. M. Vollmar and K. von Schwarzenberg, OncoTargets Ther., 2016, 8, 9576.

10 B. Luong, R. Schwenk, J. Bräutigam, R. Müller, D. Menche, I. Bischoff and R. Fürst, PLoS One, 2018, 13, e0203053. 
11 For alternative approaches to synthesizing the archazolid conjugated triene see: (a) D. Menche, J. Hassfeld, J. Li and S. Rudolph, J. Am. Chem. Soc., 2007, 129, 6100; (b) P. A. Roethle, T. C. Ingrid and D. Trauner, J. Am. Chem. Soc., 2007, 129, 8960; (c) Z. Huang and E.-i. Negishi, J. Am. Chem. Soc., 2007, 129, 14788; (d) S. M. Swick, S. L. Schaefer and G. W. O'Neil, Tetrahedron Lett., 2015, 56, 4039; (e) S. Scheeff and D. Menche, Org. Lett., 2019, 21, 271.

12 Glycosylation at either the C7- or C15-hydroxyls (archazolids $\mathrm{C}$ and $\mathrm{E}$ respectively) significantly reduces their V-ATPase inhibitory activity, see: N. Horstmann, S. Essig, S. Bockelmann, H. Wieczorek, M. Huss, F. Sasse and D. Menche, J. Nat. Prod., 2011, 74, 1100.

13 M. E. Mowery and P. DeShong, J. Org. Chem., 1999, 64, 1684.

14 G. C. Fortman and S. P. Nolan, Chem. Soc. Rev., 2011, 40, 5151.

15 E. A. Crane, T. P. Zabawa, R. L. Farmer and K. A. Scheidt, Angew. Chem., Int. Ed., 2011, 50, 9112.

16 S. E. Kelly, in Comprehensive Organic Synthesis, ed. B. M. Trost and I. Fleming, Pergamon, Oxford, 1991, vol. 1, pp. 729-817.

17 A. K. Mandal, J. S. Schneekloth, K. Kuramochi and C. M. Crews, Org. Lett., 2006, 8, 427.

18 R. E. Boer, J. A. Giménez-Bastida, O. Boutaud, J. Somnath, C. Schneider and G. A. Sulikowski, Org. Lett., 2018, 20, 4020. 19 N.-W. Tseng and M. Lautens, J. Org. Chem., 2009, 74, 2521.
20 K. Endo, M. Hirokami and T. Shibata, J. Org. Chem., 2010, 75, 3469.

21 J. R. Coombs, L. Zhang and J. P. Morken, Org. Lett., 2015, 17, 1708.

22 S. Namirembe, C. Gao, R. P. Wexler and J. P. Morken, Org. Lett., 2019, 21, 4392.

23 J. Gorges and U. Kazmaier, Org. Lett., 2018, 20, 2033.

24 D. Menche, H. Irschik, F. Sasse, A. Raja and M. Altendorfer, Org. Biomol. Chem., 2013, 11, 2116.

25 R. Noyori and T. Ohkuma, Angew. Chem., Int. Ed., 2001, 40, 40.

26 B. M. Trost and D. Laurent, Chem. Sci., 2016, 7, 4985.

27 For a recent review see: A. T. K. Koshvandi and M. M. Heravi, Appl. Organomet. Chem., 2018, 32, e4210.

28 A. B. Tran, G. Melly, R. Doucette, B. Ashcraft, L. Sebren, J. Young and G. W. O'Neil, Org. Biomol. Chem., 2011, 9, 7671.

29 L. B. Smart, F. Vojdani, M. Maeshima and T. A. Wilkins, Plant Physiol., 1998, 116, 1539.

30 D. Reker, A. M. Perna, T. Rodrigues, P. Schneider, M. Reutlinger, B. Mönch, A. Koeberle, C. Lamers, M. Gabler, H. Steinmetz, R. Müller, M. Schubert-Zsilavecz, O. Werz and G. Schneider, Nat. Chem., 2014, 6, 1072.

31 A COX activity assay kit was purchased from Cayman Chemical, https:/www.caymanchem.com/product/760151, accessed 8/5/2019). See ESI† for details. 